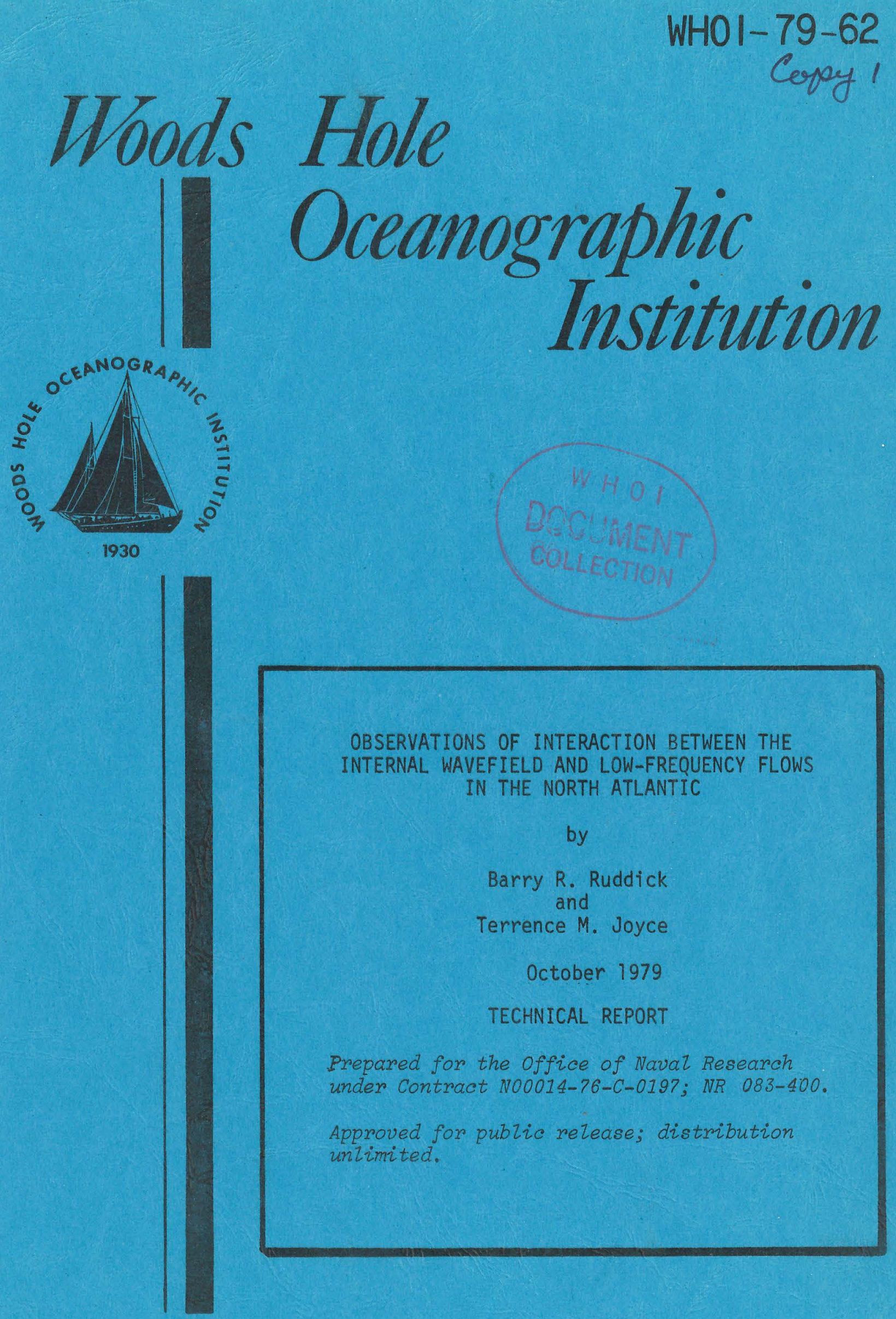

WOODS HOLE, MASSACHUSETTS 02543 
WHOI $-79-62$

\title{
OBSERVATIONS OF INTERACTION BETWEEN THE INTERNAL WAVEFIELD AND \\ LOW-FREQUENCY FLOWS IN THE NORTH ATLANTIC
}

\author{
by
}

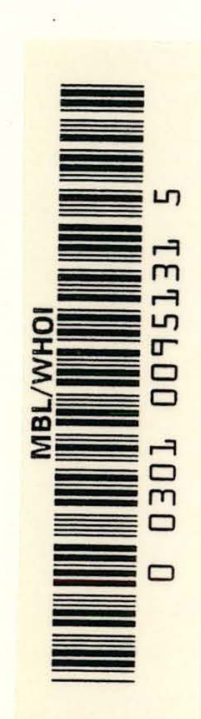

\author{
Barry R, Ruddick \\ and \\ Terrence M. Joyce \\ WOODS HOLE OCEANOGRAPHIC INSTITUTION \\ Woods Hole, Massachusetts 02543 \\ October 1979
}

TECHNICAL REPORT

Prepared for the Office of Naval Research under Contract N00014-76-C-0197; NR 083-400.

Reproduction in whole or in part is permitted for any purpose of the United States Goverment. In citing this report in a bibliography, the reference given should be to "Journal of Physical Oceanography, Vol. 9, No. 3, May 1979, pp. 498-517".

Approved for public release; distribution unlimited.

Approved for Distribution: Valentine Worthington, Chairman Department of Physical Oceanography 
Reprinted from Journal of Physical Oceanography, Vol. 9, No. 3, May 1979

American Meteorological Society

Printed in U. S. A.

\title{
Observations of Interaction between the Internal Wavefield and Low-Frequency Flows in the North Atlantic
}

\author{
BARRY R. RUDDICK \\ Research School of Earth Sciences, Institute of Advanced Studies, \\ The Australian National University, Canberra ACT Australia \\ TERRENCE M. Joyce \\ Woods Hole Oceanographic Institution, Woods Hole, MA 02543
}

(Manuscript received 26 April 1978, in final form 15 November 1978)

\begin{abstract}
A total of four moorings from POLYMODE array I and II were analyzed in an investigation of the interaction of wavefields and mean flow. In particular, evidence for internal wave-mean flow interaction was sought by searching for time correlations between the vertically acting Reynolds stress of the wavefield (estimated using the temperature and velocity records), and the mean shear. No significant stress-shear correlations were found at the less energetic moorings $\left(\bar{u} \leqslant 10 \mathrm{~cm} \mathrm{~s}^{-1}\right)$, indicating that the magnitude of the eddy viscosity was under $200 \mathrm{~cm}^{2} \mathrm{~s}^{-1}$, with the sign of the energy transfer uncertain. This is considerably below the $\mathrm{O}\left(4500 \mathrm{~cm}^{2} \mathrm{~s}^{-1}\right)$ predicted by Müller (1976). An extensive error analysis indicates that the large wave stress predicted by the theory should have been observable clearly under the conditions of measurement. At moorings typified by a higher mean velocity $(\bar{u} \approx 25$ $\mathrm{cm} \mathrm{s}^{-1}$ ), statistically significant stress-shear correlations were found, and the wavefield energy level was observed to modulate with the strength of the mean shear. The observations were consistent with generation of short ( $\sim 1 \mathrm{~km}$ horizontal wavelength) internal waves by the mean shear near the thermocline, resulting in an effective eddy viscosity of $\sim 100 \mathrm{~cm}^{2} \mathrm{~s}^{-1}$.

Theoretical computations indicate that the wavefield "basic state" may not be independent of the mean flow as assumed by Müller (1976) but can actually be modified by large-scale vertical shear and still remain in equilibrium. In that case, the wavefield does not exchange momentum with a large-scale vertical shear flow and, excepting critical-layer effects, a small vertical eddy viscosity is to be expected. Using the Garrett-Munk (1975) model internal wave spectrum, estimates were made of the maximum momentum flux (stress) expected to be lost to critical-layer absorption. This stress was found to increase almost linearly with the velocity difference across the shear zone, corresponding to a vertical eddy viscosity of $-100 \mathrm{~cm}^{2} \mathrm{~s}^{-1}$. Stresses indicative of this effect were not observed in the data.
\end{abstract}

\section{Introduction}

Natural internal waves have been observed in water for some time now (cf. Murray, 1892) and even longer in the atmosphere. However, it has only been in recent years that the observations have been quantitatively interpreted. Present day oceanic internal wave measurements can be interpreted in a common framework, the $\omega$-k spectral model of Garrett and Munk (1972, hereafter GM72). [Two updated versions are Garrett and Munk (1975, 1976; hereafter GM75 and GM76) described in Desaubies (1976) and Cairns and Williams (1976).] These models bridge the gap between theory and observation, enabling the prospective experimenter to ask in advance of a particular experiment, "Am I likely to see anything that gives new information?" and get an answer. This awareness of which information was missing has prompted the development of many new ways to measure the internal wavefield (cf. Briscoe, 1975a). These measurements have tightened up knowledge of the frequency and wavenumber shape of the universal spectrum to a high degree, and allowed inferences to be made about the cause and nature of the mechanisms which shape the spectrum.

While major inroads are beginning to be made regarding the internal shaping processes of the spectrum, Wunsch (1975a) has pointed out that there are major gaps in the knowledge of the relative importance of various possible sources and sinks for internal wave energy. One potentially important source (or sink) of energy is interaction with the (usually) larger scale, low-frequency flow, which tends to be more energetic than the internal wave- 
field and so at least has a lot of energy to give to the wavefield. Whether the low-frequency flow gives or takes energy on the average, and how rapidly, are the primary questions asked in this work.

Most theoretical studies of propagation of internal waves in the presence of a mean shear current (cf. Garrett, 1968) indicate that an internal wave can propagate through a vertical shear flow with no exchange of momentum unless the wave is dissipated, such as at a critical level (Booker and Bretherton, 1967). A scenario in which internal lee waves are generated at the lower boundary of the atmosphere, propagate upward and are dissipated at a critical level is described by Bretherton (1969b). The net effect is the distribution of the topographic drag over a much greater depth, which can be described in terms of a kinematic viscosity. A similar computation has been performed for the ocean by Bell (1975) who estimates a vertical momentum flux of $0.5 \mathrm{~cm}^{2} \mathrm{~s}^{-2}$ (more propertly, dyn $\mathrm{cm}^{-2}$ ) for a bottom current of $5 \mathrm{~cm} \mathrm{~s}^{-1}$. Dissipation of the energy of these waves at a critical level would result in a vertical mixing coefficient of order $10 \mathrm{~cm}^{2}$ $\mathrm{s}^{-1}$ at the level of dissipation.

Müller $(1976,1977)$ has studied the interaction of a field of internal gravity waves with a large-scale mean flow. Using the concept of an equilibrium spectral shape (with that of Garrett and Munk in mind), Müller investigates a balance between the tendency of the mean shear flow to distort the spectrum and the internal relaxation tendencies of the wavefield, which tend to restore the equilibrium shape. Assuming that this equilibrium shape is independent of the mean flow, Müller finds that the balance of tendencies (distortion and relaxation) lead to asymmetries in the wavefield such that the field exerts a stress which opposes the mean shear. The interaction may, in a local limit, be parameterized by eddy viscosities, both horizontal $\left[\mathrm{O}\left(10^{5}\right) \mathrm{cm}^{2} \mathrm{~s}^{-1}\right]$ and vertical $\left[\mathrm{O}\left(10^{3}\right) \mathrm{cm}^{2} \mathrm{~s}^{-1}\right]$. Because of the large aspect ratio of the mean flow, the vertical eddy viscosity is more important than the horizontal to the energy balance of the wavefield. Müller estimates that the interaction results in an energy exchange rate of $10^{-5} \mathrm{erg} \mathrm{cm}^{-3} \mathrm{~s}^{-1}$ from the mean flow to the wavefield. This is probably the dominant source of energy for the internal wavefield if the Müller interaction is as strong as predicted. An eddy viscosity this large will cause decay times for baroclinic motions comparable to those observed for Gulf Stream rings, so the interaction may also be of importance to the mean flow.

Observations of internal wave-mean flow interaction are somewhat sparse. Frankignoul $(1974,1976)$ discovered in internal wave records a tendency for the wave velocities to be more intense in a direction perpendicular to a strong mean current than parallel to it. He tentatively identified critical-layer wave absorption as a possible cause for this effect. $\mathrm{He}$ also found that the horizontal anisotropy of the currents was related to the large-scale mean shears, in a manner consistent with some of the predictions of the Müller theory. However, Frankignoul was only able to verify that horizontal variations in the medium (i.e., the shear flow) will distort the wavefield; this is a propagation effect. Direct estimation of the horizontal eddy viscosity effects predicted by Müller proved impossible with the data at hand, because of the relatively short data length and the high statistical noise level of the horizontally acting wave stress. Frankignoul found that the internal wavefield energy level and the magnitude squared of the mean vertical shear were correlated, suggesting an energy exchange; however, the hypothesized energy exchange mechanism (vertical eddy viscosity) was inferred and not directly observed.

The central point of the observations presented here is a direct test of the vertical eddy viscosity aspect of the Müller (1976) interaction. Using horizontal current and temperature data from longterm POLYMODE array I and II moorings in the North Atlantic, estimates of the vertically-acting Reynolds stresses of the wavefield and low-frequency vertical shear (often referred to as "mean" because it appears so to the wavefield) were estimated as slowly varying time series. Statistical correlation analyses were performed to see if the wavefield has any tendency to exert stresses which oppose or reinforce the mean shear, thereby extracting energy from or giving it up to) the shear. To the extent that Reynolds stresses can be accurately estimated using temperature records, the observations of momentum exchange with the shear flow are direct.

The effect of the Coriolis force on horizontal wave velocities couples the momentum and buoyancy fluxes exerted by a wave. The effect of these fluxes on a quasi-geostrophic mean flow is discussed in Section 2, where it is argued that the near-inertial frequency waves contribute little to the vertical flux of vorticity, and so should be eliminated from the estimate of "effective" wave stress. In Section 3 the data and their analysis are described, and the observations are presented in Section 4.

A major finding in Section 4 is that the interaction between the wavefield and a vertical shear flow must be much weaker than predicted by Müller. In Section 5, the Müller interaction theory is discussed, and an extremely simple equilibrium wave field solution is presented. This equilibrium state is "flexible" in the sense that it can be modified by the mean shear, and except for the effects of critical-level absorption, does not exchange momentum with the mean flow. It is argued that an equilibrium field with similar balances (and which is non-interacting) may exist in the ocean. 
2. Momentum and buoyancy fluxes in a geostrophic shear flow

The effect of internal waves on a mean flow is measured by the divergence of the momentum and buoyancy flux tensors which the wavefield sets up. In the presence of rotation, the momentum and buoyancy fluxes of the wavefield are related, and combine in the vorticity equation to form a single "effective" stress tensor. This stress tensor can be rather simply related to the actual stress tensor and turns out to be easier to estimate accurately than the actual stress. At near-inertial frequencies, the momentum and buoyancy fluxes cancel in their effect on a geostrophic flow and so do not contribute to the "effective" stress. For this reason, an accurate estimate of the "effective" stress is obtained by integrating the cospectrum $C_{\alpha 3}$ from $2 f$ to $N$ (i.e., by eliminating the frequency range $f-2 f$ from the integration).

As discussed in Rhines (1973), ocean current spectra nearly always exhibit a relative lack of energy (a "gap") at periods between one and ten days. Because of this, the data may be uniquely separated into high-frequency (inertial frequency and above) and low-frequency (below inertial frequency) components. The high-frequency components are consistent with internal wave dynamics (Fofonoff, 1969). Müller (1976) decomposes the Eulerian equations of motion into separate, coupled sets of equations for the mean (low-frequency) flow and for the wavefield. (Because of the spectral gap, space-time averages may be replaced by time averages.) The equations for the mean flow are

where

$$
\begin{gathered}
\left(\frac{\partial}{\partial t}+\overline{\mathbf{u}} \cdot \nabla\right) \bar{u}_{i}+\epsilon_{i 3 k} f \bar{u}_{k}-\bar{b} \delta_{i 3}+\frac{1}{\rho_{0}} \frac{\partial \bar{p}}{\partial x_{i}}=-\frac{\partial}{\partial x_{j}} F_{i j}, \\
\left(\frac{\partial}{\partial t}+\overline{\mathbf{u}} \cdot \nabla\right) \bar{b}+N^{2} \bar{u}_{3}=-\frac{\partial}{\partial x_{j}} M_{j},
\end{gathered}
$$

$$
\begin{array}{ll}
F_{i j} & \text { mean wave-induced momentum flux }\left[=\overline{u_{i}^{\prime} u_{j}^{\prime}}+\bar{P} w \delta_{i j}\right] \\
M_{j} & \text { mean wave-induced buoyancy flux }\left[=\overline{b^{\prime} u_{j}^{\prime}}\right] \\
\bar{u}_{i} & \text { Eulerian mean velocity } \\
u_{i}^{\prime} & \text { Eulerian perturbation velocity } \\
\bar{b} & \text { mean buoyancy field }\left\{=-\left(g / \rho_{0}\right)\left[\bar{\rho}(\mathbf{x}, t)-\rho_{e}\right]\right\} \\
b^{\prime} & \text { perturbation buoyancy field }\left\{=-\left(g / \rho_{0}\right)\left[\rho^{\prime}(\mathbf{x}, t)-\rho_{e}\right]\right\} \\
\rho_{e}\left(x_{3}\right) & \text { equilibrium density stratification } \\
N^{2}\left(x_{3}\right) & \text { equilibrium buoyancy frequency }\left[=-\left(g / \rho_{0}\right) \partial \rho_{e} / \partial x_{3}\right] .
\end{array}
$$

The pressure has been decomposed into four parts: 1) the hydrostatic pressure associated with the equilibrium stratification; 2 ) the mean pressure field associated with the mean flow $(\bar{p}) ; 3)$ the mean waveinduced pressure $\left(\bar{p}_{w}\right)$; which is steady on the short time scale; 4$)$ and the perturbation wave-induced pressure $\left(p_{w}^{\prime}\right)$, which has zero mean.

The effect of the wave field on the mean flow is given by the divergence of the various wave-induced fluxes on the right-hand side of the equations. If the fluxes are linearly related to certain mean flow gradients,

where

$$
\begin{aligned}
& F_{\alpha \beta}=-\nu_{H} \frac{\partial \bar{u}_{\alpha}}{\partial x_{\beta}} \quad\left(\nu_{H}=\text { horizontal eddy viscosity }\right), \\
& F_{\alpha 3}=-\nu_{v} \frac{\partial \bar{u}_{\alpha}}{\partial x_{3}} \quad\left(\nu_{v}=\text { vertical eddy viscosity }\right), \\
& M_{\alpha}=-D_{H} \frac{\partial \bar{b}}{\partial x_{\alpha}} \quad\left(D_{H}=\text { horizontal eddy diffusivity }\right),
\end{aligned}
$$

$$
M_{3}=0 \text { for internal waves. }
$$

The indices $i, j$ vary over $1-3 ; \alpha, \beta$ vary over 1,2 . Then the mean flow equations are closed, and the effects of the wavefield on the mean flow are similar to the analogous molecular viscosity and diffusivity effects. The theoretical analysis by Müller (1976) suggests that in the limit of a local interaction, the relations above should be observable, with

$$
\begin{aligned}
\nu_{H} & \approx 7 \times 10^{4} \mathrm{~cm}^{2} \mathrm{~s}^{-1}, \\
\nu_{v} & \approx 4 \times 10^{3} \mathrm{~cm}^{2} \mathrm{~s}^{-1}, \\
D_{H} & \approx-2 \times 10^{4} \mathrm{~cm}^{2} \mathrm{~s}^{-1} .
\end{aligned}
$$

The vertical eddy viscosity predicted by Müller is two orders of magnitude larger than the values 
typically used in ocean circulation models, and so the effect may be important to both the mean flow and the wavefield. It is the observational verification of this aspect of the interaction that is the primary concern of this paper.

\section{a. The "effective" wave stress}

The low frequency motions are, to a first approximation, geostrophically balanced (the MODE-I Dynamics Group, 1975). As discussed by Müller (1976), the equation of motion for a quasigeostrophic flow is the potential vorticity equation. The effects of a field of internal waves on the quasi-geostrophic flow appear in the vorticity equation as

$$
-\epsilon_{\alpha \beta 3} \frac{\partial}{\partial x_{\alpha}} \frac{\partial}{\partial x_{j}} T_{\beta j},
$$

where

$$
T_{\alpha j}=F_{\alpha j}-\epsilon_{\alpha \beta 3} \frac{f}{N^{2}} M_{\beta} \delta_{j 3} .
$$

So, in terms of their effect on a geostrophically balanced mean flow, the vertically acting momentum flux and horizontal buoyancy flux appear together, in what can be termed an effective stress. The buoyancy flux might be thought of as the ability to tilt the isopleths of the mean flow field, and through the thermal wind relation, affect the mean shear in a perpendicular direction. Jones (1967) has shown $T_{\alpha 3}$ to be proportional to the vertical flux of angular momentum of the wave, while Bretherton (1969b) has identified $T_{\alpha 3}$ as the vertical flux of horizontal momentum across a material surface.

\section{b. The relation between Reynolds stress and "effec- tive" stress}

The equations of motion for the fluctuating field are (Müller, 1976)

$$
\begin{aligned}
& \frac{D u_{i}^{\prime}}{D t}+ f \epsilon_{i 3 k} u_{k}^{\prime}-b^{\prime} \delta_{i 3}+\frac{1}{\rho_{0}} \frac{\partial p^{\prime}}{\partial x_{i}} \\
&+u_{j}^{\prime} \frac{\partial}{\partial x_{j}} \bar{u}_{i}=-\frac{\partial}{\partial x_{j}}\left(u_{i}^{\prime} u_{j}^{\prime}-\overline{u_{i}^{\prime} u_{j}^{\prime}}\right), \\
& \frac{D b^{\prime}}{D t}+N^{2} u_{3}^{\prime}+u_{j}^{\prime} \frac{\partial \bar{b}}{\partial x_{j}}=-\frac{\partial}{\partial x_{j}}\left(u_{j}^{\prime} b^{\prime}-\overline{u_{j}^{\prime} b^{\prime}}\right)
\end{aligned}
$$

where

$$
\frac{D}{D t}=\frac{\partial}{\partial t}+\overline{\mathbf{u}} \cdot \nabla
$$

The terms on the right-hand side represent the effects of wave-wave interactions. These are a higher order effect and will be ignored here. Since the horizontal scale of the mean velocity field $(\sim 100 \mathrm{~km})$ is much larger than the horizontal scale of oceanic internal waves $(\approx 10 \mathrm{~km})$, the concept of a wave which is locally plane in the horizontal is valid (WKB in the horizontal direction, to lowest order). Note that no assumptions about vertical scales are necessary as long as $\bar{u}_{3}$ is small. In what follows we shall drop the primes which denote the wave variables.

We rotate the coordinate system so that the $x$, axis is aligned with the horizontal wavenumber component of a single wave $[\cos (k, x,-\omega t)$ dependence]. The equation for the cross-wavenumber velocity is then

$$
\frac{D u_{2}}{D t}+f u_{1}=0 .
$$

Defining quasi-Lagrangian horizontal particle displacements, $\zeta_{\alpha}$ by

$$
\frac{D \zeta_{\alpha}}{D t}=u_{\alpha}, \quad \overline{\zeta_{\alpha}}=0,
$$

then it can easily be shown from (2.11) that

$$
\zeta_{1}=-\frac{u_{2}}{f}, \quad \zeta_{2}=\frac{f}{\omega_{0}{ }^{2}} u_{1},
$$

where $\omega_{0}=\omega-\mathbf{k} \cdot \overline{\mathbf{u}}$ (intrinsic frequency).

We can now relate the buoyancy fluxes, $M_{j}$ of the wave to the momentum fluxes $F_{i j}$ :

$$
\begin{aligned}
M_{2} & =\overline{u_{2} b} \\
& =\frac{\overline{D \zeta_{2}}}{D t} b \\
& =-\zeta_{2} \frac{D b}{D t} \\
& =\frac{f N^{2}}{\omega_{0}{ }^{2}} \overline{u_{1} u_{3}}+\frac{f}{\omega_{0}{ }^{2}}\left(\overline{u_{1}{ }^{2}} \frac{\partial \bar{b}}{\partial x_{1}}+\overline{u_{1} u_{2}} \frac{\partial \bar{b}}{\partial x_{2}}\right) .
\end{aligned}
$$

The two terms on the right in curly brackets were estimated from current meter observations (Ruddick, 1977) and found to be negligible compared to the first term, as they must be for self-consistency of the WKB approximation. Thus,

$$
\begin{aligned}
& M_{2}=\frac{f N^{2}}{\omega_{0}^{2}} F_{13} . \\
& M_{1}=-\frac{N^{2}}{f} F_{23} .
\end{aligned}
$$

Note that the relationship between $F_{13}$ and $M_{2}$ is different from the relationship between $F_{23}$ and $M_{1}$. We can divide the wave into two parts: one involved in vertical propagation of "effective" momentum (ray) and one which is not (mode).

The part of the wave associated with $F_{13}$ is raylike. The effective stress exerted by this part of the wave is 


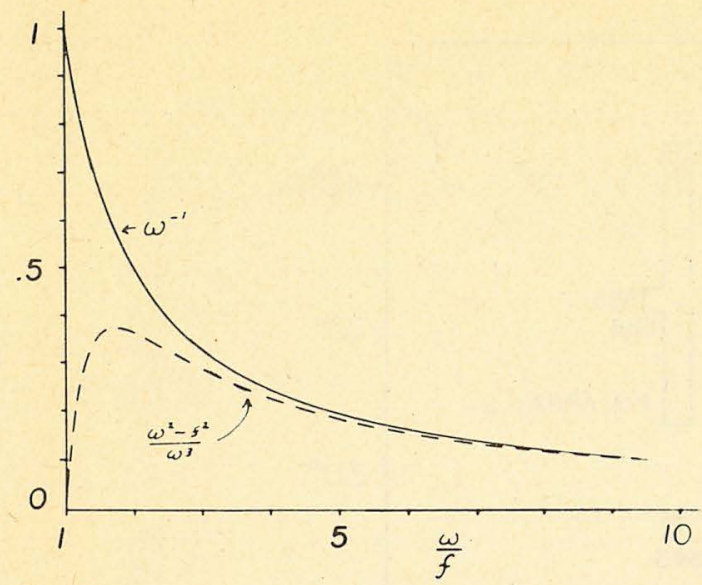

FIG. 1. Actual (solid line) and effective (dashed line) vertical flux of horizontal momentum as a function of frequency, estimated from the Garrett and Munk (1975) model internal wave spectrum.

$$
\begin{aligned}
T_{13} & =F_{13}-\frac{f}{N^{2}} M_{2} \\
& =F_{13}\left(1-\frac{f^{2}}{\omega_{0}^{2}}\right) .
\end{aligned}
$$

This part of the wave is associated with vertical phase propagation, vertical propagation of energy (Jones, 1967), and in the case of a plane wave, is called a ray.

The part of the wave associated with $M_{1}$ is modelike. The effective stress exerted by this part of the wave is

$$
\begin{aligned}
T_{23} & =F_{23}+\frac{f}{N^{2}} M_{1} \\
& =0 .
\end{aligned}
$$

Hence the vertical flux of cross-wavenumber momentum $F_{23}$ is not associated with a vertical flux of vorticity. This part of the wave has the properties of a mode (i.e., no vertical energy flux) and is characterized by a mass flux $M_{1}$ in the direction of the wavenumber. The momentum flux $F_{23}$ is due to the effect of the Coriolis force on the horizontal velocities, and represents a body force, $\partial F_{23} / \partial x_{3}$, which oscillates rapidly with depth, integrating to zero. ( $F_{23}=0$ at top, bottom and one or more intermediate depths.) The theoretical formulation of Müller (1976) does not allow modes unless the orthogonality conditions [Müller, 1976, Eq. (2.37)] are altered to account for the phase-locking of upward and downward waves (Ruddick, 1977).

The multi-wave generalization of (2.20) gives an estimate of the effective wave stress

$$
T_{\alpha 3}=\int_{f}^{N} d \omega C_{\alpha 3}(\omega)\left(1-\frac{f^{2}}{\omega^{2}}\right)
$$

where $C_{\alpha 3}$ is the cospectrum between $u_{\alpha}$ and $u_{3}$. We have approximated $\omega_{0} \approx \omega$ due to lack of information about the Doppler shift $\mathbf{k} \cdot \mathbf{u}$. In the Appendix, we show that this term is small for waves comprising the Garrett and Munk (1975) spectrum, but we see in Section 4 that the Doppler effect is important for some waves observed in addition to that spectrum. In estimating the effective stress this way, we are ignoring the "noise" stress associated with the presence of modes. The cospectral density predicted by the Garrett and Munk (1975) model internal wave spectrum if the wave field were fully anisotropic is

$$
\left(\overline{u_{1}^{2}} \overline{u_{3}^{2}}\right)^{1 / 2}=\frac{2 E_{0} f}{\pi^{2} N} \omega^{-1},
$$

where $E_{0}=30 \mathrm{~cm}^{2} \mathrm{~s}^{-2}$. The frequency dependence of the above cospectrum (solid line) and the quantity $C_{\alpha 3}(\omega)\left(1 f^{2} / \omega^{2}\right)$ (dashed line) are plotted in Fig. 1. The two integrands differ primarily near the inertial frequency. A fair estimate of $T_{\alpha 3}$ is attained using

$$
\int_{2 f}^{N} d \omega C_{\alpha 3}(\omega)
$$

(i.e. by eliminating the frequency range $f-2 f$ from the integration). For the frequency dependence shown, this estimate is only $7 \%$ below the effective stress. The frequency range eliminated is strongly dominated by inertial oscillations, which do not contribute to the interaction, and the semidiurnal tidal motions, which are beyond the scope of the Müller theory. We denote the wave stress integrated over the frequency range $2 f-N$ as the "continuum band" stress; this is a fair estimate of the effective wave stress. The more conventional estimate, integrated from $f$ to $N$, is called the "total band" stress.

\section{Data description and analysis}

The data used were a subset of the moored current and temperature timeseries collected for the POLYMODE array I and array II experiments. POLYMODE is a joint U.S.-U.S.S.R. project whose scientific objectives are to study the kinematics and dynamics of low-frequency motions ("eddies") in the North Atlantic Ocean. The Array I and II measurements were designed to study the geographical variation of kinematic properties of the eddy field, such as the strength and time scale. The location of the arrays and of the four moorings used in this study are shown in Fig. 2. The southern moorings, 545 and 543 , tended to have relatively slow currents: typically $5 \mathrm{~cm} \mathrm{~s}^{-1}$ or less. The two northerly moorings, 565 and 566 , were in a region of stronger currents: typically $25 \mathrm{~cm} \mathrm{~s}^{-1}$.

At each mooring, observations of temperature 


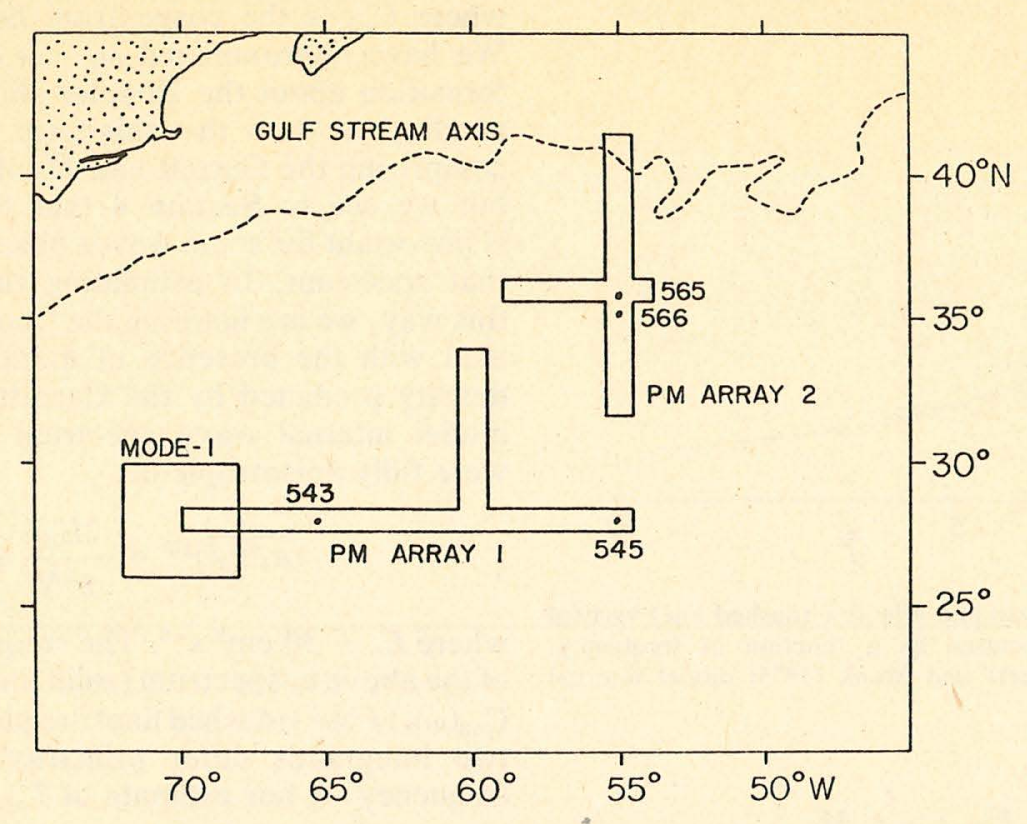

FIG. 2. Location in the North Atlantic Ocean of the moorings analyzed.

and horizontal current were taken every $15 \mathrm{~min}$ at several depths throughout the deep ocean. (To avoid surface wave contamination, the top of the moorings were at least $500 \mathrm{~m}$ below the ocean surface.) The resulting time series were typically 220 days in duration, generally containing information about several eddies. The primary measuring instrument used was the VACM (Vector Averaging Current Meter ${ }^{1}$ ), which records a vector average of the current velocity measured during the preceding 15 min period.

\section{a. Mean shear estimation}

The depths used for the current meters are shown in Fig. 3 ( $1 \mathrm{db}$ pressure corresponds to $\sim 1 \mathrm{~m}$ depth). Also shown is a realization of the low-frequency shear profile measured by Sanford (1975) in the MODE-1 region (see Fig. 2). The east component is on the upper left, the north is on the lower left. Recent computations (Sanford, personal communication, 1976) on this profile and on an independent profile indicate that $\sim 95 \%$ of the current shear is in the first baroclinic mode which is shown to the right of the shear profiles for comparison. The best simple estimate of the thermocline shear is from a first difference of the uppermost and $1000 \mathrm{~m}$ mean (low-pass-filtered) velocities. Owens and McWilliams (1976, personal communication) estimate the rms error in shear measurement to be $20 \%$ for instruments at the above depths. Assuming a local wave-

\footnotetext{
${ }^{1}$ Model 605, American Machine and Foundry, Alexandria, VA 22314
}

mean flow interaction, we expect the strongest mean shear, and so the largest wave stresses, to occur in the vicinity of the $1000 \mathrm{~m}$ instrument.

\section{b. Stress estimation}

The "wave" time series were obtained by subtracting the low-passed time series from the original series and then analyzed using a method similar to that used by McWilliams (1972) and Frankignoul and Strait (1972). The data were broken into short pieces of $75 \mathrm{~h}$ length, which overlapped by $\sim 50 \%$. (The time origin of piece $n+1$ was $37 \mathrm{~h}$ later than that of piece $n$.) Each piece was then fast Fourier transformed under a Hanning data window (full cosine arch) which was applied as a convolution filter in the frequency domain. The window allows a time resolution of $\sim 50 \mathrm{~h}$, and is essential for preventing spectral leakage from the energetic low frequencies to the higher frequencies. The vertical velocity was estimated in frequency space by assuming the temperature variations are primarily due to vertical advection

$$
u_{3}=-\Gamma^{-1} \partial T / \partial T
$$

where the mean temperature gradient estimate is

$$
\Gamma=\frac{\partial \bar{T}}{\partial x_{3}}=C_{1}+C_{2} \bar{T},
$$

an exponential fit to the mean temperature profile. The $\overline{u_{\alpha} u_{3}}$ stress was estimated by integrating the $\alpha, 3$ cospectrum over the "continuum" $(2 f-N)$ and "total" $(f-N)$ internal wave frequency bands. 

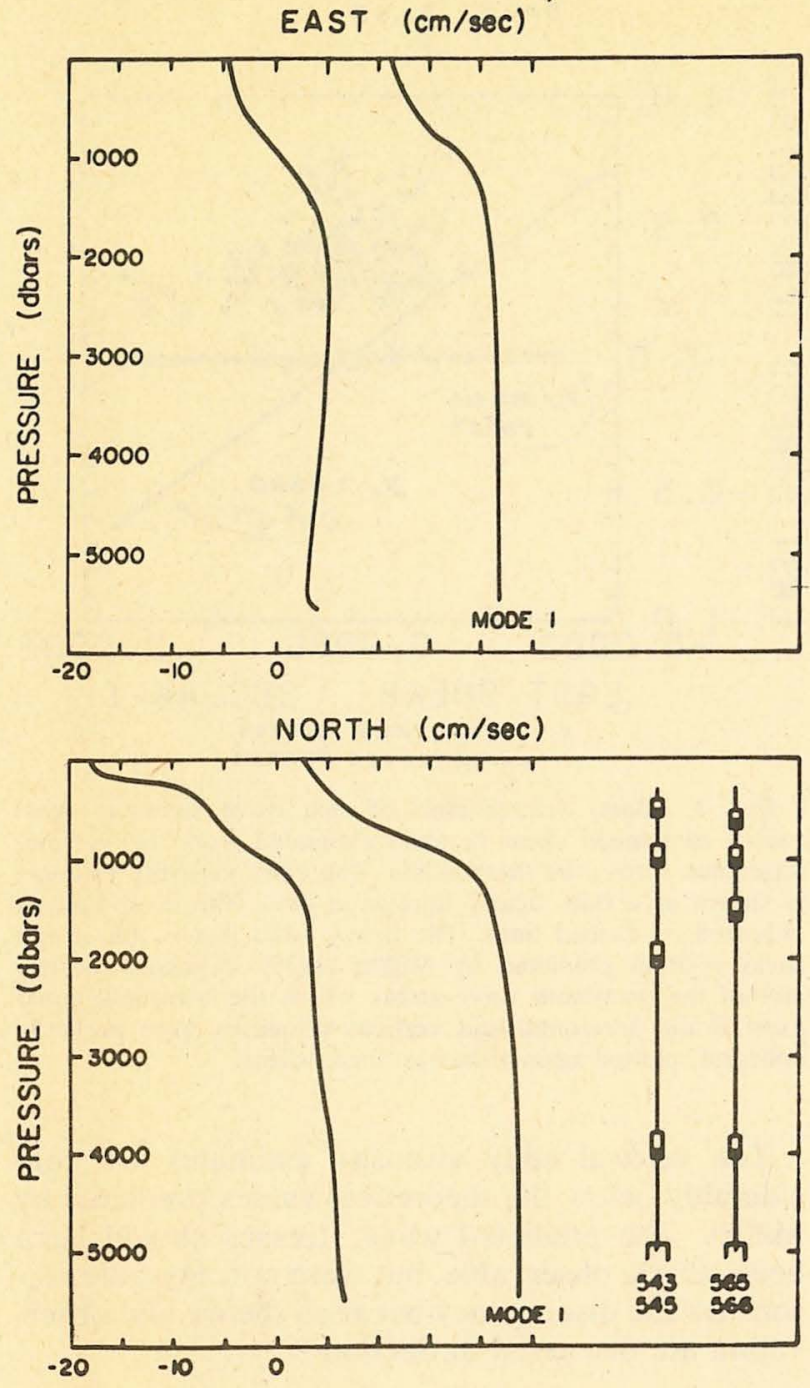

FIG. 3. Profile of baroclinic mean velocity obtained by Sanford (1975) at left, with best-fit first baroclinic mode, at right, for comparison. The depths of the current meters on the moorings which were analyzed are shown at the lower right.

Details of the filtering, windowing and Fourier transforming operations are given in Ruddick (1977). As a "by-product" of the analysis, the autospectra and cross-spectra of all other pairs of velocity components were available as slowly-varying time series.

\section{c. Errors in stress estimation}

Surprisingly, the largest single error in a wave stress estimate is the random error associated with the fact that the stress estimate is a sample of a stochastic process. A rough estimate of the standard deviation of the stress estimates about the true value is given by Jenkins and Watts (1969)

$$
\delta F \approx\left(2 \bar{u}_{1}^{2} \bar{u}_{3}^{2} / n\right)^{1 / 2} \approx 0.06 \mathrm{~cm}^{2} \mathrm{~s}^{-2},
$$

where $n=300$ is the number of points in the data piece. This estimate is rough, since it is based on the assumption that $u_{1}$ and $u_{3}$ are uncorrelated white-noise processes. However, the two effects (Ruddick, 1977) arising from $u_{1}$ and $u_{3}$ being nonwhite tend to cancel, so the estimate is at least correct in order of magnitude.

The remaining errors in estimating the wave stress in this manner arise through the use of the simplified heat equation to estimate the vertical velocity

$$
u_{3}=-\frac{\partial T}{\partial t} / \Gamma .
$$

These errors have been estimated in the Appendix. The estimated random (shear-independent) errors are $0.08 \mathrm{~cm}^{2} \mathrm{~s}^{-2}$, while the systematic errors (proportional to shear) could affect the eddy viscosity estimated by $\pm 38 \mathrm{~cm}^{2} \mathrm{~s}^{-1}$. Thus only stress observations with statistically significant regressions and estimated eddy viscosities $\mathrm{O}\left(100 \mathrm{~cm}^{2} \mathrm{~s}^{-1}\right)$ or greater can be considered as meaningful.

\section{Results}

The observations from moorings 543 and 545 ("quiet" moorings) are discussed separately from the more energetic moorings (565 and 566) because the former satisfy the conditions for validity of the Müller theory. At the latter moorings, the mean currents are higher than allowed in the Müller theory, and Doppler effects are likely to be important.

\section{a. Moorings 543 and 545 (slow mean current)}

\section{1) Stress-SHear Relations}

The vertically-acting wave stresses were computed from temperature and current measurements as described in the previous section, integrated over the total internal wave frequency band $(f-N)$ to estimate the total wave stress, and integrated over the "continuum" band $(2 f-N)$ to estimate the "effective" stress. The stress time series were compared with the mean shear to check for a stress-shear relationship of the form

$$
F_{\alpha 3}=a+b \frac{\partial \bar{u}_{\alpha}}{\partial x_{3}}+\text { (noise). }
$$

Müller (1976) estimates that $b\left(=\nu_{v}\right) \approx 4000 \mathrm{~cm}^{2}$ $\mathrm{s}^{-1}$ for the oceanic internal wavefield. The regression was performed allowing $a=0$ and $a \neq 0$, with insignificant change to the results. The uncertainty of the slope $b$ was computed following Lyttkens (1963) using a method which allows for non-whiteness and slight deviations from normality in the time series.

A typical regression (mooring 543) is shown in 
Fig. 4, in which the dots show a scatterplot of continuum band stress ( $y$ axis) versus mean shear $(x$ axis). The slope of the regression is shown by the thin solid line, and the scatter of the dots about this line corresponds with the estimated stress error $\left(\sim 0.06 \mathrm{~cm}^{2} \mathrm{~s}^{-2}\right)$. The estimated error bounds for the regression are shown as dashed lines. For comparison, the regression predicted by Müller (1976) (decreased by $7 \%$ to allow for "continuum band" underestimation) is shown as a heavy black line. From the relatively small scatter of the data, it is apparent that the stresses predicted by Müller would have been clearly observable had they occurred-the "signal" would have been clearly observable above the "noise". The observed stresses were an order of magnitude smaller than predicted by Müller. The regression in Fig. 4 yields

$$
b=29 \pm 112 \mathrm{~cm}^{2} \mathrm{~s}^{-1} \text {. }
$$

All the regressions of this sort (continuum and total bands) at moorings 545 and 543 were consistent with $\nu_{v}=0 \pm 200 \mathrm{~cm}^{2} \mathrm{~s}^{-1}$.

Also shown in Fig. 4, as crosses, are the continuum band estimates of $\left(u_{1}^{2} \frac{2}{u_{3}{ }^{2}}\right)^{1 / 2}$, the lower half of the $u_{1}, u_{3}$ coherence. This is the absolute maximum stress the wavefield could exert if $u_{1}$ and $u_{3}$ were perfectly coherent. It is readily seen that there is enough energy (if the wavefield were made asymmetric by the Müller interaction) to enable the wavefield to exert the predicted stresses. Only for shear in excess of $\sim 10^{-4} \mathrm{~s}^{-1}$ would the wavefield find it impossible to exert the predicted stresses without becoming more energetic.

\section{2) OTHER REgRESSIONS}

The kinetic energy of the internal wavefield was also estimated as a function of time and compared with the mean speed and the shear intensity. There were no changes coherent with the mean flow, which is consistent with the small observed values of eddy viscosity. In a more general search for wavemean flow correspondence, regressions were attempted which involved 1) other shear estimates (i.e., 500-1000 m); 2) mean velocities; 3) average of 500 and $1000 \mathrm{~m}$ stresses; 4) stress divergence; and 5) "total band" stress estimates. All regressions were negative. In addition, the horizontal mean density gradients were estimated from the vertical shear (assuming geostrophy), and compared with the "total band" horizontal buoyancy flux. From this, a horizontal diffusivity of

$$
D_{H}=0 \pm 6 \times 10^{+5} \mathrm{~cm}^{2} \mathrm{~s}^{-1}
$$

was estimated. Note that the error bars are an order of magnitude larger than the diffusivity predicted by Müller, so no conclusions about the diffusivity can be drawn.

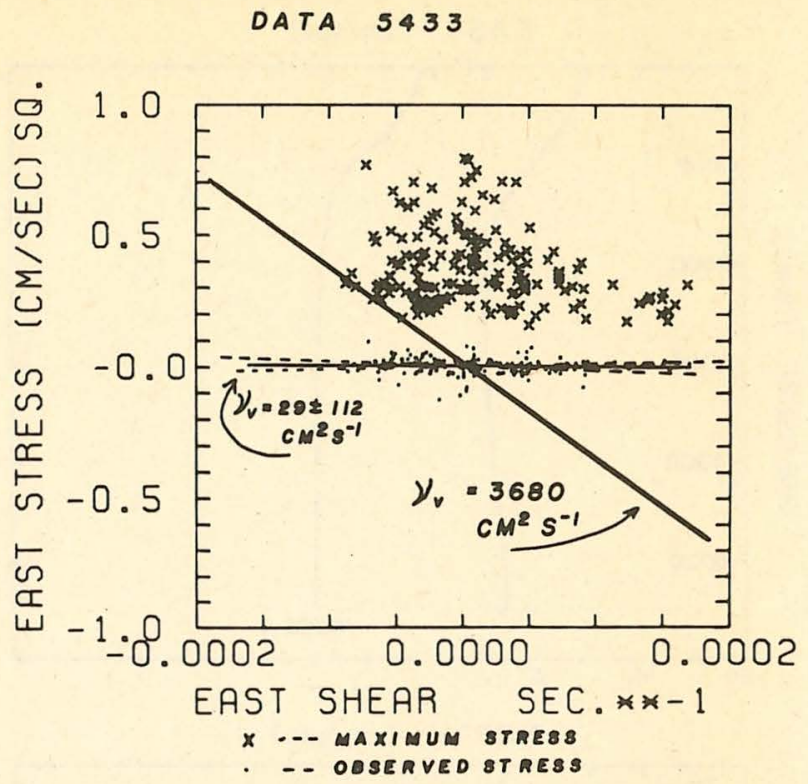

FIG. 4. (Dots) Scatter plots of east wave stress ( $y$ axis) versus east mean shear ( $x$ axis) estimated from the velocity difference across the thermocline. The eddy viscosity estimate is shown as a thin, nearly horizontal line, with error bounds indicated as dashed lines. The heavy solid line is the stressshear relation predicted by Müller (1976). (Crosses) Scatter plot of the maximum wave stress which the wavefield could exert if the horizontal and vertical velocities were perfectly coherent, plotted against the east mean shear.

The vertical eddy viscosity estimates are considerably below the theoretical values predicted by Müller. The predicted wave stresses should have been clearly observable, but were not. Possible reasons for the discrepancy between theory and observation are discussed in Section 5.

\section{b. Moorings 565 and 566 (fast mean current)}

The mean velocity at these moorings was typically $\sim 30 \mathrm{~cm} \mathrm{~s}^{-1}$ of the order of the phase speed of an internal wave. The expansion parameter $\delta \omega / \omega$ in the Müller theory was not small at these moorings, so the theory is not strictly valid. We also expect Doppler shift and associated effects (such as critical layers) to be important.

\section{1) Stress-mean Flow correlations}

Fig. 5 shows scatterplots of the continuum band north-vertical wave stress versus the north component of the mean velocity, from mooring 565 . The upper plot is the stress at $600 \mathrm{~m}$ depth, and the lower plot is the stress at $1000 \mathrm{~m}$ depth, both plotted against the $600 \mathrm{~m}$ mean velocity. The $600 \mathrm{~m}$ stress is positively correlated with the velocity, while the $1000 \mathrm{~m}$ stress is negatively correlated with the velocity. Both correlation coefficients are strongly significant, and a similar result holds at 

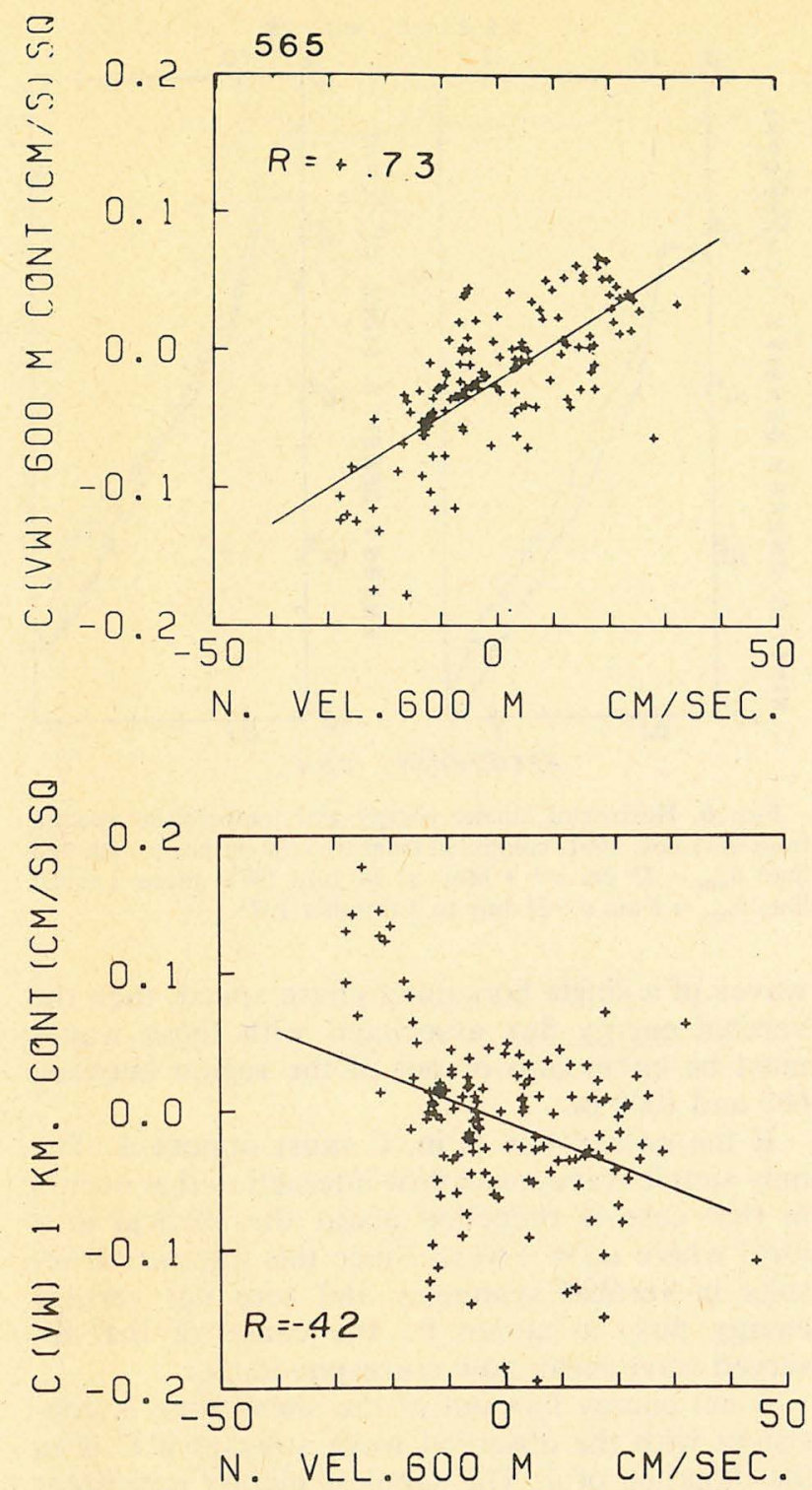

Fig. 5. Scatter plots of north wave stress ( $y$ axis) versus $600 \mathrm{~m}$ north mean velocity ( $x$ axis) at mooring 565. Upper plot: $600 \mathrm{~m}$ stress versus $600 \mathrm{~m}$ velocity. Lower plot: $1000 \mathrm{~m}$ stress versus $600 \mathrm{~m}$ velocity. $R$ is the correlation coefficient obtained from the regression of stress on velocity.

mooring 566. (Correlation coefficients exceeding 0.35 are significant at the $95 \%$ confidence level.) In all cases, the stresses are more strongly correlated with the mean velocity than with the mean shear. Regression against the shear results in eddy viscosity estimates

$$
\begin{aligned}
& \text { (Estimated) } \nu_{v} \\
& =\left\{\begin{array}{l}
-100 \mathrm{~cm}^{2} \mathrm{~s}^{-1} \text { at } 600 \mathrm{~m} \text { depth }\left( \pm 50 \mathrm{~cm}^{2} \mathrm{~s}^{-1}\right) \\
+100 \mathrm{~cm}^{2} \mathrm{~s}^{-1} \text { at } 1000 \mathrm{~m} \operatorname{depth}\left( \pm 90 \mathrm{~cm}^{2} \mathrm{~s}^{-1}\right)
\end{array}\right.
\end{aligned}
$$

The change in sign with depth is not consistent with ideas of a local eddy viscosity nor with the ob- served changes in wavefield energy level, an example of which is presented below.

The mean flow at mooring 566 exhibited a nonstationarity on a long (several months) time scale. For the first third of the 200-day record, the mean velocities were strong, typically $25 \mathrm{~cm} \mathrm{~s}^{-1}$, and the shear was typically $1.7 \times 10^{-4} \mathrm{~s}^{-1}$. For the second third, they were $\sim 6 \mathrm{~cm} \mathrm{~s}^{-1}$ and $4 \times 10^{-4} \mathrm{~s}^{-1}$. In Fig. 6 are shown two sets of spectra, with horizontal kinetic energy on the left and temperature on the right. The frequencies resolved cover the internal wave frequency band from $0.04 \mathrm{cph}$ (near the inertial frequency) to $2 \mathrm{cph}$ (near the buoyancy frequency). The spectra computed using data from the first third of the record, when the mean velocities were quite high, are shown in dashes. The spectra computed using data from the second third of the record, when the mean velocity was slower, are shown as solid curves. Note that both the kinetic and potential energy levels of the wave-field decreased by a factor of 2 in association with the factor of 20 decrease in the mean kinetic energy. The wave energies increased again with the shear for the final third (not shown.) The changes are most pronounced at the higher internal wave frequencies. The particular data shown are from the $600 \mathrm{~m}$ level on mooring 566 .

At both moorings 565 and 566, and at both the 600 and $1000 \mathrm{~m}$ levels, the wavefield energy level was found (by means of regression methods) to vary with the intensity of the mean velocity and shear.

The observed energy level changes at $600 \mathrm{~m}$ depth are inconsistent with the observed wave stresses at that level. The rate of working of the wave stresses against the mean shear,

$$
\begin{aligned}
\frac{d E}{d t} \text { wave } & =-\left(\overline{u_{\alpha} u_{3}}\right) \partial \bar{u}_{\alpha} / \partial x_{3} \\
& =\nu_{v}\left|\partial \overline{\mathbf{u}} / \partial x_{3}\right|^{2},
\end{aligned}
$$

shows that energy is transferred from the wavefield to the mean flow at the $600 \mathrm{~m}$ level if the wave stresses are as observed. When the mean shear increases, the wavefield energy level ought to decrease, but the opposite is observed. It is suspicious that this inconsistency should occur at the $600 \mathrm{~m}$ level, where mean velocities tend to be the strongest. This leads one to suspect that Doppler effects, discussed below, are affecting these observations. We now mention a few simple rules relating the vertical energy flux, and the true and temperature-observed momentum fluxes of a single internal wave. These will be helpful in interpreting the above observations.

2) Doppler effects on the stress estimates

As in Section 3, we assume a wavelike dependence proportional to exp $-i\left(k_{1} x_{1}+k_{2} x_{2}-\omega t\right)$. 
Then from Eq. (3.4), allowing for the Dopplershift term, we have from Eq. (3.12)

$$
F_{\alpha 3}^{M}=F_{\alpha 3}^{T} /(1-\mathbf{k} \cdot \overline{\mathbf{u}} / \omega),
$$

where subscription $M$ indicates the stress observed using the temperature. Hence the Doppler shift can change the sign of the stress, as observed using temperature, from its true value according to $\mathbf{k}: \overline{\mathbf{u}} \gtrless \omega$. We view this wave as an additional signal to the isotropic background spectrum, since in the Appendix we showed that Doppler effects on the background spectrum are weak.

Jones (1967) investigated the propagation of horizontally plane waves in a geostrophic vertical shear flow. From his equations (his $\mathbf{k}$ is minus our k) it is easy to show

$$
\overline{p u_{3}}=\frac{\omega_{0}}{k_{\alpha}}\left(\overline{u_{\alpha} u_{3}}+\epsilon_{\beta \alpha 3} \overline{\zeta_{\beta} u_{3}}\right),
$$

(no $\alpha$ summation; $\alpha$ either 1 or 2 )

where $\zeta_{\beta}$ is defined by (2.15) as a Langrangian particle displacement. From Eqs. (2.17) and (2.18) we obtain, considering vertically propagating waves,

$$
\begin{aligned}
& \overline{p u_{3}}=\frac{\omega_{0}}{k_{\alpha}} F_{\alpha 3}^{T}\left(1-\frac{f^{2}}{\omega_{0}^{2}}\right), \\
& \overline{p u_{3}}=\frac{\omega_{0}^{2}-f^{2}}{k_{\alpha} \omega} F_{\alpha 3}^{M} .
\end{aligned}
$$

\section{(again, no $\alpha$ summation)}

Note that the above relations are for a single wave and do not allow a unique interpretation of the sign of the dominant energy flux or phase speed of many waves. A field of waves with both upward and downward propagating waves can give rise to a Reynolds stress of either sign, with the net energy flux and momentum flux not simply related. It is possible to find more than one interpretation that is consistent with the observations. We shall look for the simplest one, hypothesizing waves with a single, dominant wave phase speed.

Suppose that the waves which are causing the observed stresses at the 600 and $1000 \mathrm{~m}$ levels have the same horizontal phase speed. We define coordinates such that the mean shear is in the $x_{1}$ direction; then we have observed $F_{13}^{M}>0$ at $600 \mathrm{~m}$ and $F_{13}^{M}<0$ at $1000 \mathrm{~m}$, correlated with the mean shear. Denoting $C=\omega / k_{1}$ as the component of phase speed in the $x_{1}$ direction, Eq. (4.9) becomes for $\alpha=1$

$$
\overline{p u_{3}}=\frac{\omega_{0}^{2}-f^{2}}{k_{1}^{2}} \frac{F_{13}^{M}}{C} .
$$

Then, since $C$ is assumed the same at both levels, $\overline{p u_{3}}$ must have opposite signs at 600 and $1000 \mathrm{~m}$. In other words, if the observed stresses are caused by

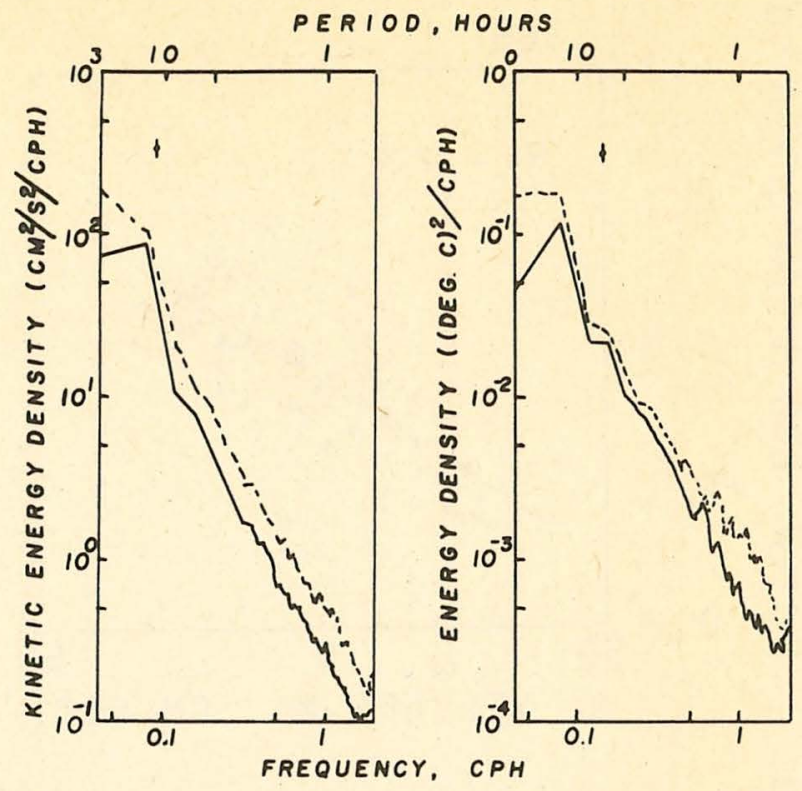

FIG. 6. Horizontal kinetic energy and temperature spectra from data nos. 5661, computed from two times: piece 1 (dotted line) $\bar{u}_{\mathrm{rms}}=25 \mathrm{~cm} \mathrm{~s}^{-1} 9$ May to 24 July 1975; piece 2 (solid line) $\bar{u}_{\mathrm{rms}}=6 \mathrm{~cm} \mathrm{~s}^{-2} 23$ July to 2 October 1975 .

waves of a single horizontal phase speed, then the vertical energy flux associated with these waves must be either into or out of the region between 600 and $1000 \mathrm{~m}$.

If the energy flux is in, $C$ must oppose $\bar{u}$. The only simple wave-mean flow interaction that occurs in that case is reflection about the vertical at a level where $\omega_{0}=+N(z)$. Since this interaction results in vertical symmetry and zero net vertical energy flux, it cannot be the cause of the observed wave-mean flow correspondences.

A net energy flux out of the shear zone is consistent with the observed wave stresses if $C$ is in the direction of $\bar{u}$. The simplest picture consistent with both the energy and stress observations is one of internal wave generation at the region of maximum shear, located between the 600 and $1000 \mathrm{~m}$ levels. For generation to occur, the shear Richardson number must be below 0.25 , and yet the large-scale Richardson number was rarely below 50. There are three ways in which wave generation may still occur and each of them may be important in the ocean:

1) Shear-finestructure interaction: Stewart (1969) and Scorer (1969) argued that the shear tends to be concentrated at regions of high density gradient, such that the local Richardson number is much smaller than the large-scale value. Scorer goes on to describe how the density structure can be sustained by interaction between the shear and the finestructure.

2) The shear can be enhanced by the (intermit- 

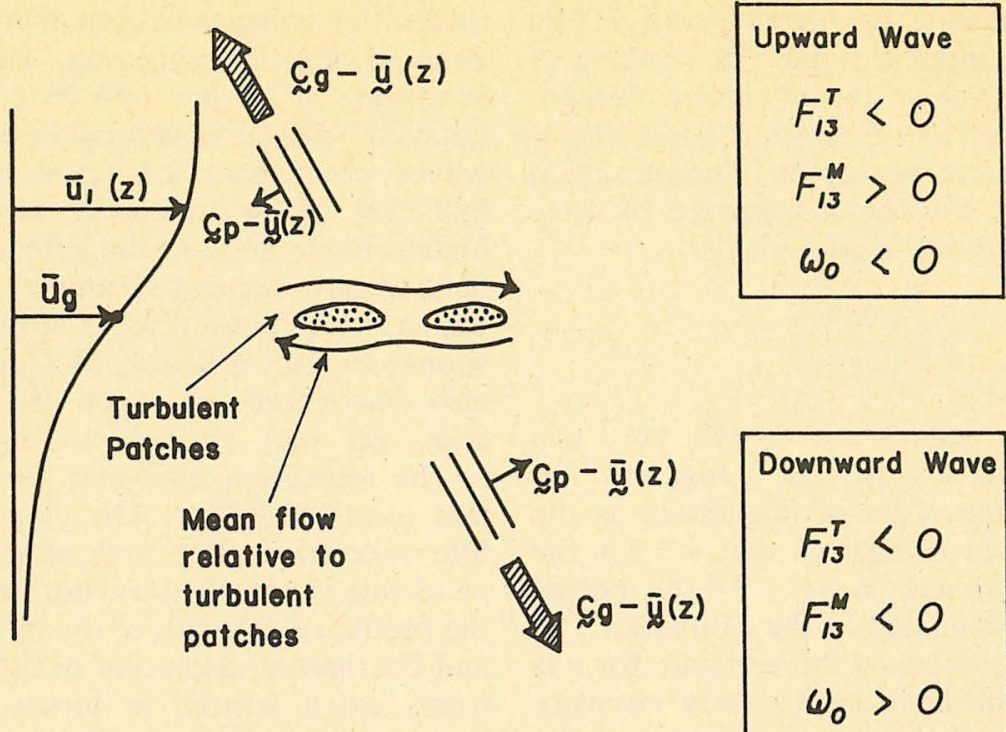

FIG. 7. Sketch of the wave generation process causing the observed wave stress-mean flow correlations. The shear flow relative to turbulent patches of water generates "lee" waves traveling at the advection speed $U_{g}$ of the patches. Both the upward and downward traveling waves exert a negative Reynolds stress, transferring momentum downward. However, the upward traveling wave is advected faster than its horizontal phase speed $U_{g}$, causing severe Doppler effects. These cause a positive Reynolds stress to be inferred from the velocity and temperature records.

tent) presence of inertial oscillations. There are hints in the observations of enhanced wave generation associated with inertial "events."

3) Neutrally stable "cat's-eye" billows (overturning regions), maintained by the shear flow (Maslowe, 1973) may cause a disturbance to the mean flow, leading to generation of internal waves in much the same manner as generation of lee waves by flow over obstacles.

Each of the above mechanisms leads either to Kelvin-Helmholtz billows or patches of turbulence which are advected by the mean flow. The shear flow relative to these will generate internal waves which propagate vertically away from the disturbances. The generated waves will have horizontal phase speed equal to the speed of advection of the generating disturbances. We now consider in detail the hypothesis of internal wave generation at the shear zone.

A picture of the wave generation process which is consistent with the observations is the following. We consider a positive $\bar{u}_{1}(z)$, increasing upward and denote the mean velocity at the level of maximum shear by $U_{g}$. At this level, the shear causes and/or maintains turbulent patches which are advected at speed $U_{g}$. The shear flow relative to the patches cause "lee" waves to be generated which are stationary in a frame of reference moving at $U_{g}$ (see Fig. 7). If we adopt the sign convention that $\omega$ is positive, then $k_{1}=\omega / U_{g}$ is also positive.
The wave with downward energy flux (negative $\overline{p u_{3}}$ ) has positive intrinsic frequency, since $\omega_{0}$ $=\omega-k_{1} \bar{u}_{1}(z)$ and $\bar{u}_{1}(z)<U_{g}$. From Eqs. (4.8) and (4.9), the wave exerts a negative Reynolds stress and a negative stress is measured. The wave which travels upward is in a region where $\bar{u}_{1}(z)$ $>U_{g}$ and has negative intrinsic frequency. From (4.8) the stress exerted by the wave is negative, but from (4.9) the stress measured is positive. The Doppler effects in the temperature measurement have changed the sign of the measured stress for this wave, since the advection velocity is greater than the horizontal phase speed. Note that both the upward and downward traveling waves exert a negative Reynolds stress, thus effecting a transfer of mean momentum from above the shear zone to below it.

Since the mean velocity tends to be about twice the velocity at maximum shear, then by Eq. (4.6) the magnitude of the observed stress is about the same as the true value, and so we estimate

$$
\nu_{v} \approx+100 \mathrm{~cm}^{2} \mathrm{~s}^{-1} \text { at } 600 \mathrm{~m} \text { and } 1000 \mathrm{~m} \text {, }
$$

i.e., both the upward and downward waves transfer positive momentum downward. The negative eddy viscosity estimate (4.4) is in error because of the Doppler effect.

The positive eddy viscosity estimate at $600 \mathrm{~m}$ is consistent with the observations of wavefield energy increase with shear intensity. A wavefield 
energy model suggested by Frankignoul (1976) balances the energy input through the working of the wave stresses against the shear by dissipation processes modeled as $-E / \tau$, where $\tau$ is a relaxation time for dissipative processes. Thorpe (1975) estimates $\tau \sim 9$ days. For the changes $\Delta E$ of wave energy level observed, the model yields

$$
\Delta E \sim \nu_{v} \tau\left|\frac{\partial \overline{\mathbf{u}}}{\partial x_{3}}\right|^{2} .
$$

Substitution of the values $\nu_{v}=+100 \mathrm{~cm}^{2} \mathrm{~s}^{-1}$, $\tau=10$ days, $\left|\partial \overline{\mathbf{u}} / \partial x_{3}\right|^{2}=10^{-7} \mathrm{~s}^{-1}$ suggests $\Delta E$ $=9 \mathrm{~cm}^{2} \mathrm{~s}^{-2}$, the same order of magnitude as the observed energy level change $\left(9 \mathrm{~cm}^{2} \mathrm{~s}^{-2}\right.$ for the continuum frequency band, $26 \mathrm{~cm}^{2} \mathrm{~s}^{-2}$ if the inertial frequencies are also included in the estimate.) The calculation is inexact because the estimate for $\tau$ is only approximate, and because the eddy viscosity may be underestimated due to attenuation of the wave stresses before the waves reach the observation levels.

The temperature spectrum in Fig. 6 shows that the wave energy level changes were greatest at periods of 1 to $8 \mathrm{~h}$. This is, in fact, substantiated by performing the stress-mean velocity regression using stress estimated as a function of wave frequency (the $u_{1}-u_{3}$ cospectrum), in which the correlation is strongest at the above periods. Taking a typical $U_{g}$ of $10 \mathrm{~cm} \mathrm{~s}^{-1}$, we can estimate the length scales of the generated waves. Setting $C$ $=\omega / k=10 \mathrm{~cm} \mathrm{~s}^{-1}$, and substituting $\omega$ corresponding to 1-8 $\mathrm{h}$ periods in Eq. (4.7), we find that the horizontal wave lengths are between $400 \mathrm{~m}$ and 3 $\mathrm{km}$, somewhat shorter than the dominant waves in the Garrett-Munk spectra.

\section{On the theory of wavefield-mean flow interactions}

As discussed in Section 4a, the conditions for validity of the Müller theory were satisfied at moorings 545 and 543 , and the mean shear was sufficiently strong that the predicted wave stresses should have been clearly observable above the noise level. Yet no wave stress-mean shear correlations were observed, leading to the conclusion that the vertical eddy viscosity is at least a factor of 20 smaller than the predicted value. Under conditions of shear so intense that a correlated stress signal is observed, the observations are inconsistent with the predictions of the Müller theory. Whatever interaction occurs between the internal wave-field and the low-frequency flow is both different in nature and weaker than the interaction predicted by Müller.

In their investigation of some of the detailed effects of nonlinear interactions among a spectrum of internal waves, McComas and Bretherton (1977) touch on several possible reasons for the Müller interaction being weaker than predicted. One effect, denoted elastic scattering, describes the backscattering of a wave into its counterpart with the opposite vertical wavenumber by inertial frequency waves which have a vertical wavelength equal to half that of the scattered wave. At frequencies higher than inertial, the effect forces the wavefield toward vertical symmetry, and so attenuates any vertical fluxes of wave momentum set up in response to a mean shear. McComas and Bretherton also discovered that some of the relaxation processes act very strongly to maintain equilibrium, so the relaxation time may be much shorter than that used by Müller. The choice of a representative relaxation time and of its wavenumber dependence is not obvious, but has a direct effect on the predicted strength of the interaction. McComas and Bretherton also point out that the GM76 spectrum, when written in terms of action density, has no vertical wavenumber gradient over much of the domain, and so in the Müller formalism should not be affected by a vertical shear. However, Müller shows that the vertical wavenumber dependence in the interaction integrates out, becoming independent of the distribution of wave energy in wavenumber space. This is true even for a spectrum which is white over part of the wavenumber range, and so one would expect that the Müller interaction should still occur.

An assumption implicit in the Müller theory is that the equilibrium wavefield, used as the basic state for a perturbation expansion, is independent of the mean flow, so that shear induced perturbations to the wavefield will tend to relax to the original $(\bar{u}=0)$ spectral shape. By using a particular source term (induced diffusion), which McComas and Bretherton have indicated dominates much of the spectrum, we will show by example that it is conceivable for the equilibrium wave state to depend on the mean velocity through the dispersion relation. This somewhat subtle difference drastically alters the strength and sense of the predicted momentum exchange, leading to a zero or negative vertical eddy viscosity, rather than the large positive value predicted by Müller. Therefore, the predictions of the interaction theory are very sensitive to the details of the processes which maintain the equilibrium spectrum.

\section{a. The radiation balance equation}

The basis for the theory of interaction between the internal wavefield and a larger scale mean flow is the radiation balance equation (Müller and Olbers, 1975). The equation governs the time, space and wavenumber evolution of the wave action density spectrum, $\eta(k, x, t)$

where

$$
\mathscr{L}(\eta)=\mathscr{I}(\eta)
$$




$$
\begin{array}{rlrl}
\mathscr{L} & =\frac{\partial}{\partial t}+\dot{\mathbf{x}} \frac{\partial}{\partial x}+\dot{\mathbf{k}} \frac{\partial}{\partial \mathbf{k}} & & \text { Liouville operator } \\
\dot{\mathbf{x}} & =\frac{\partial \Omega}{\partial \mathbf{k}} & & \text { group velocity } \\
\dot{\mathbf{k}} & =-\frac{\partial \Omega}{\partial \mathbf{x}} & & \text { rate of refraction } \\
\Omega(\mathbf{k}, \mathbf{x}, t) & =\Omega_{0}(\mathbf{k}, \mathbf{x}, t)+\mathbf{k} \cdot \overline{\mathbf{u}} & & \\
& =\left(\frac{N^{2}\left(x_{3}\right)\left(k_{1}{ }^{2}+k_{2}{ }^{2}\right)+f^{2} k_{3}{ }^{2}}{k_{i} k_{i}}\right)+\mathbf{k} \cdot \overline{\mathbf{u}} & & \text { dispersion relation } \\
\omega_{0} & =\omega-\mathbf{k} \cdot \overline{\mathbf{u}} & & \text { intrinsic frequency } \\
\eta & =\epsilon / \omega_{0} & & \text { wave action density } \\
\epsilon(\mathbf{k}, x, t) & & \text { wave energy density. }
\end{array}
$$

The Liouville operator on the left side of (5.1) comes from the conservation law for wave action (Bretherton and Garrett, 1968) applied to a random field of waves. The source term $\mathscr{S}$ represents, in a statistical sense, the increase or decrease in wave action density due to interaction with other waves, interaction with external fields, dissipation, etc. $\mathscr{T}$ is a catch-all for the nonconservative processes which affect the wave in its travels. In his study of the effects of wave-wave interactions, Olbers (1976) introduced a valuable concept which characterises the source term in a simple way: the equilibrium spectrum is determined by statistically irreversible processes such as resonant interactions, and deviations from this state will decay irreversibly toward equilibrium at a rate predetermined by the details of the process. The time scale of this decay is the relaxation time $\tau_{R}$.

\section{b. The Müller theory}

For the purposes of later discussion, we briefly describe the assumptions and the perturbation expansion used by Müller (1977).

Müller regarded the solution of the radiation balance equation in the absence of mean flow as the equilibrium state $\eta_{0}$. He expanded the problem in a series, regarding as perturbations all changes in the solution and operators which are due to the mean flow

$$
\begin{aligned}
\mathscr{L} & =\mathscr{L}_{0}+\delta \mathscr{L}, \\
\eta & =\eta_{0}+\eta_{1}+\ldots, \\
\mathscr{S} & =\mathscr{S}\left(\eta_{0}\right)+\frac{\delta \mathscr{S}}{\delta \eta}\left[\eta_{1}+\ldots\right],
\end{aligned}
$$

where

$$
\mathscr{L}_{0}=\frac{\partial}{\partial t}+\frac{\partial \Omega_{0}}{\partial \mathbf{k}} \cdot \frac{\partial}{\partial \mathbf{x}}-\frac{\partial \Omega_{0}}{\partial \mathbf{x}} \cdot \frac{\partial}{\partial \mathbf{k}},
$$

$$
\delta \mathscr{L}=\bar{u}_{i} \frac{\partial}{\partial x_{i}}-k_{j} \frac{\partial \bar{u}_{j}}{\partial x_{i}} \frac{\partial}{\partial k_{i}} .
$$

The lowest order balance

$$
\mathscr{L}_{0}\left(\eta_{0}\right)=\mathscr{P}\left(\eta_{0}\right)
$$

gives the equilibrium state, regarded as known. The problem at first order is

$$
\mathscr{L}_{0}\left(\eta_{1}\right)+\delta \mathscr{L}\left(\eta_{0}\right)=\frac{\delta \mathscr{S}}{\delta \eta}\left[\eta_{1}\right] .
$$

At this point, Müller made a relaxation time approximation

$$
\frac{\delta \mathscr{S}}{\delta \eta} \approx-\tau_{R}{ }^{-1} .
$$

The effect of this assumed form of the source term

$$
\frac{\delta \mathscr{S}}{\delta \eta} \eta_{1} \approx-\frac{\eta-\eta_{0}}{\tau_{R}}
$$

is to cause the spectrum to relax to the original $(\bar{u}=0)$ spectrum with time scale $\tau_{R}$. Müller then finds that the balance between mean flow induced perturbations to the propagation characteristics (distorting the spectrum) and the above relaxation effects cause wave stresses which oppose the shear. Since the predicted eddy viscosities vanish as $\tau_{R} \rightarrow \infty$, the relaxation effects are necessary for the interaction to occur. Because of the form of the perturbation source term (5.8), Müller has implicitly assumed that the equilibrium state is independent of the mean flow, and that relaxation effects will drive the spectrum toward this state, even in the presence of shear. However, if the equilibrium state can depend on the shear flow through the dispersion relation, the shear-altered spectrum is by definition in equilibrium, and so the relaxation 
term (5.8) is absent. In this case, the interaction is much weaker. We will now demonstrate that such a "flexible" equilibrium state is possible.

\section{c. A simplified alternative theory}

We will consider the greatly simplified problem of a wavefield in a simple shear flow $\bar{u}\left(x_{3}, t\right)$, so that the dependence on $x_{1}$ and $x_{2}$ drops out of the problem. For a particular form for the relaxation term $S$, we will show the following:

- The wavefield described by the solution to the radiation balance equation (5.1) for a steady mean flow $\bar{u}\left(x_{3}\right)$ has nondivergent momentum flux, and so is in equilibrium with the mean flow. This noninteracting state is in contrast to the interaction predicted by Müller for the same situation.

- The solution will continually adjust to a slowly varying mean flow, $\bar{u}\left(x_{3}, t\right)$ remaining nearly in equilibrium. Since the deviation from equilibrium is much smaller than that envisaged in the Müller theory, the interaction is much weaker.

The particular relaxation mechanism we will use is from McComas and Bretherton (1977), who showed that over much of the internal wave spectrum ( $\left.\omega \geqslant 2 f, k \geqslant 10 \mathrm{~km}^{-1}\right)$, the dominant effect of wave-wave interactions is represented by a diffusion of wave action in vertical wavenumber space

$$
\mathscr{S}=\frac{\partial}{\partial k_{3}}\left(a_{33} \frac{\partial \eta}{\partial k_{3}}\right),
$$

where

$$
a_{33}=2(10)^{-5} \mathrm{~s}^{-1}\left|\frac{\omega}{f} \frac{k_{3}}{k_{3}^{*}}\right| k_{3}{ }^{2}
$$

is the diffusion coefficient, and $k_{3}^{*}=6 \mathrm{~km}^{-1}$ is the vertical wavenumber scale for the GM75 model. The internal waves have been approximated by McComas and Bretherton as hydrostatic $\left(\omega^{2} \ll N^{2}\right)$, and for this mechanism the inequality $\omega^{2} \gg f^{2}$ is true. Therefore, a valid approximation to the dispersion relation is

$$
\omega=\Omega(\mathbf{k})=N\left(k_{1}^{2}+k_{2}^{2}\right)^{1 / 2} /\left|k_{3}\right| .
$$

When written in terms of $\mathbf{k}$ and $V_{3}=\partial \Omega / \partial k_{3}$, the diffusion coefficient becomes

$$
\begin{aligned}
a_{33} & =-2(10)^{-5} \sec ^{-1}\left[N^{2} f^{-1}\left(k_{1}^{2}+k_{2}^{2}\right)\left(k_{3}^{*}\right)^{-1}\right] s V_{3}^{-1} \\
& =F\left(k_{1}, k_{2}, z\right) V_{3}^{-1},
\end{aligned}
$$

where $V_{3}$, the vertical group velocity, is

$$
\begin{gathered}
V_{3}=\frac{\partial \Omega}{\partial k_{3}}=-s \frac{N\left(k_{1}{ }^{2}+k_{2}{ }^{2}\right)^{1 / 2}}{k_{3}{ }^{2}}, \\
s=\operatorname{sign}\left(k_{3}\right) .
\end{gathered}
$$

We suppose that the source term (5.9) is the dominant one, and that the mean flow is a function only of time and $k_{3}$. Then the radiation balance equation is greatly simplified:

$$
\frac{\partial \eta}{\partial t}+\frac{\partial \Omega}{\partial k_{3}} \frac{\partial \eta}{\partial x_{3}}-\frac{\partial \Omega}{\partial x_{3}} \frac{\partial \eta}{\partial k_{3}}=\frac{\partial}{\partial k_{3}}\left(a_{33} \frac{\partial \eta}{\partial k_{3}}\right) .
$$

For a steady mean flow $\bar{u}\left(x_{3}\right)$ the coefficients involving $\Omega$ will also be steady, and so (5.12) will have a steady solution obeying the equation

$$
\begin{aligned}
\frac{\partial \Omega}{\partial k_{3}} \frac{\partial \eta}{\partial x_{3}}-\frac{\partial \Omega}{\partial x_{3}} & \frac{\partial \eta}{\partial k_{3}} \\
& =\frac{\partial}{\partial k_{3}}\left(-s \frac{F\left(k_{1}, k_{2}, x_{3}\right)}{V_{3}} \frac{\partial \eta}{\partial k_{3}}\right) .
\end{aligned}
$$

With the change of independent variables from $k_{3}, x_{3}$ to

$$
\left.\begin{array}{rl}
\omega & =\Omega\left(k_{3}, x_{3}\right) \\
z & =x_{3}
\end{array}\right\},
$$

Eq. (5.13) becomes

$$
\frac{\partial \eta}{\partial z}=-s F\left(k_{1}, k_{2}, z\right) \frac{\partial^{2} \eta}{\partial \omega^{2}} .
$$

This is a diffusion equation in which the diffusion coefficient $F$ depends on the timelike variable $z$. For well-posedness, the boundary conditions at the top should apply to downward propagating waves, and those at the bottom to upward propagating waves. This is so the boundary layers decay in the direction of wave energy propagation.

Superposing solutions obtained by separation of variables, the solution to (5.15) is (in terms of $k_{3}, x_{3}$ )

$$
\begin{aligned}
\eta= & \sum_{n} \exp \left(-s n^{2} \int d z F\right) \\
& \times\left(A_{n} \sin n \Omega+B_{n} \cos n \Omega\right)+C+D \Omega,
\end{aligned}
$$

where the various $n$ (dimensions $\omega^{-1}$ ) are separation constants chosen to form a complete set of functions (such as a Fourier series). The constants $A_{n}$, $B_{n}, C, D$ are chosen to fit the (unknown) boundary conditions. The $A_{n}$ and $B_{n}$ terms decay away from the boundaries, representing an adjustment of the wave action flux input at the top and bottom to the diffusive equilibrium solution of the oceanic interior:

$$
\eta=C+D \Omega\left(k_{3}, x_{3}\right) .
$$

Referring back to (5.15) and using (5.10), we see that the decay length of the adjustment is approximately

$$
\begin{aligned}
\omega^{2} F^{-1} & =f k_{3}^{*} /\left(2 \times 10^{-5} \mathrm{~s}^{-1} k_{3}^{2}\right) \\
& \approx 3 k_{3}^{*} / k_{3}^{2} .
\end{aligned}
$$

At $k_{3}=6 \mathrm{~km}^{-1}(1 \mathrm{~km}$ vertical wavelength), the scale is $500 \mathrm{~m}$, and the scale rapidly becomes shorter with decreasing wavelength. This is a very 
rapid adjustment to the interior equilibrium solution (5.17).

Note that the equilibrium state is flexible; it depends implicitly on the mean flow through the dispersion relation. A change in the mean flow affects the dispersion relation, the propagation characteristics, and so the equilibrium state. Because of this, any equilibrium solution of the form $\eta_{D}(\Omega)$, such as (5.17), represents a solution with a constant (with depth) vertical flux of wave action, and so has a nondivergent effective momentum flux (which is equal to the horizontal wavenumber times the action flux). To see this, we compute the divergence of the effective vertical flux of momentum

$$
\begin{aligned}
& \frac{d}{d x_{3}} T_{13} \\
& =\frac{d}{d x_{3}} \iiint d^{3} \mathbf{k} k_{1} V_{3} \eta_{D}\left(k_{1}, k_{2}, \Omega\left(\mathbf{k}, x_{3}\right), s\right) \\
& =\frac{d}{d x_{3}} \int_{-\infty}^{\infty} d k_{1} \int_{-\infty}^{\infty} d k_{2} \int_{f+\mathbf{k} \cdot \overline{\mathbf{u}}}^{N+\mathbf{k} \cdot \overline{\mathbf{u}}} d \omega \sum_{s} k_{1} \\
& \quad \times \frac{\partial \Omega}{\partial k_{3}} \eta_{D}\left(k_{1}, k_{2}, \omega, s\right) \cdot\left|\frac{\partial \Omega}{\partial k_{3}}\right|^{-1} \\
& =\frac{d}{d x_{3}} \int_{-\infty}^{\infty} d k_{1} \int_{-\infty}^{\infty} d k_{2} \int_{f+\mathbf{k} \cdot \overline{\mathbf{u}}}^{N+\mathbf{k} \cdot \overline{\mathbf{u}}} d \omega \\
& \simeq-\int_{-\infty}^{\infty} d k_{1} \int_{-\infty}^{\infty} d k_{2} k_{1} \eta_{D} s \eta_{D}\left(k_{1}, k_{2}, \omega, s\right) \\
& \quad \times\left(k_{1}, k_{2}, f+\mathbf{k} \cdot \mathbf{u},+1\right) \mathbf{k} \cdot \frac{\partial \overline{\mathbf{u}}}{\partial x_{3}} .
\end{aligned}
$$

(We have assumed vertical symmetry (reflection) at $\omega-\mathbf{k} \cdot \mathbf{u}=N$; absorption at $\omega-\mathbf{k} \cdot \mathbf{u}=f$.) The only flux divergence is due to critical-layer absorption. The strength of this effect has been estimated using the GM76 spectrum (Ruddick, 1977) to be approximately

$$
\frac{\partial T_{13}}{\partial x_{3}} \approx-V \frac{\partial \bar{u}_{1}}{\partial x_{3}},
$$

where

$$
V \approx 0.0028 \mathrm{~cm} \mathrm{~s}^{-1} \approx 2.4 \mathrm{~m} \mathrm{day}^{-1} .
$$

Based on the shear profile of Fig. 3, this stress-shear relation corresponds roughly to a vertical eddy viscosity of $-100 \mathrm{~cm}^{2} \mathrm{~s}^{-1} . V$ is also the velocity at which the mean shear profile would move downward due to wave momentum absorption, if all other effects were absent. Critical-layer absorption is a fairly weak effect. The signature of critical-layer absorption was not observed at any of the moorings studied.

Under the assumptions of the Müller theory, the steady interior solution (5.17) would be expected to exert a steady and strong back interaction on the mean shear. Except for critical layer effects, ignored in the Müller theory, the wavefield (5.17) does not exchange energy and momentum with a steady mean flow.

However, the mean flow changes with time. The wavefield must adjust to these changes, and in so doing will interact with the mean flow. For a mean flow which changes slowly enough, the following heuristic argument will estimate the strength of the adjustment process.

Consider a slowly varying mean flow, and a wavefield in equilibrium with it. We model the changes to the mean flow by large changes $\delta \bar{u}$ $=\mathrm{O}(\bar{u})$ spaced at intervals $T \gg \tau$, where $\tau=a_{33}^{-1} k_{3}^{2}$ is the relaxation time for the wavefield. After a change $\delta \bar{u}$, the wave field decays toward the equilibrium state represented by (5.17) and approaches this state with timescale $\tau$. During this decay, the wave field interacts with the mean flow in the manner described by Müller, with $\delta \bar{u}$ replacing $\bar{u}$. The interaction stops after a few relaxation times, but the next perturbation to the mean flow is not for time $T$. We note the following:

- The average effect on the mean flow is weaker than predicted by the Müller interaction by a factor $\tau / T$, the fraction of time the interaction is occurring.

- If the mean flow stops changing the wave field will, in time $\tau$, relax to the equilibrium state (5.17). The interaction will then stop.

The mean flow actually changes smoothly and steadily on a timescale $T_{M} \gg \tau$. We then expect the solution to (5.12) to vary (after decay of initial transients) on the same time-scale. Accordingly, we expand the solution in a regular perturbation series allowing for variation on the slow time scale $T$. The small parameter used for the expansion is $\epsilon=\tau / T_{M}$. Thus,

$$
\begin{gathered}
\frac{\partial}{\partial t} \rightarrow \frac{\partial}{\partial t}+\epsilon \frac{\partial}{\partial T}+\mathrm{O}\left(\epsilon^{2}\right), \\
\eta=\eta_{0}+\epsilon \eta_{1}+\mathrm{O}\left(\epsilon^{2}\right),
\end{gathered}
$$

The problems for the lowest two orders are then

$$
\begin{gathered}
\frac{\partial \Omega}{\partial k_{3}} \frac{\partial \eta_{0}}{\partial x_{3}}-\frac{\partial \Omega}{\partial x_{3}} \frac{\partial \eta_{0}}{\partial k_{3}}-\frac{\partial}{\partial k_{3}}\left(a_{33} \frac{\partial \eta_{0}}{\partial k_{3}}\right)=0 \\
\frac{\partial \Omega}{\partial k_{3}} \frac{\partial \eta_{1}}{\partial x_{3}}-\frac{\partial \Omega}{\partial x_{3}} \frac{\partial \eta_{1}}{\partial k_{3}}-\frac{\partial}{\partial k_{3}}\left(a_{33} \frac{\partial \eta_{1}}{\partial k_{3}}\right)=-\frac{\partial \eta_{0}}{\partial T} .
\end{gathered}
$$

The zeroth-order problem (5.23) is equivalent to the problem for a steady mean flow (5.13). This simply means that for $T \gg \tau$, the wavefield sees the mean flow as steady. The lowest order solu- 
tion $\left(\eta_{0}\right)$ is given by (5.16), and by (5.17) away from boundaries. The earlier discussion for the solution (5.17) is also valid for $\eta_{0}$.

To lowest order, the wavefield is in equilibrium with the mean flow, not interacting with it. The wavefield is also "flexible" in that it continually adjusts to a changing mean flow.

The interaction occurs at first order, in the problem for $\eta_{1}$. The forcing term in (5.24) is

$$
-\frac{\partial \eta_{0}}{\partial T}=-\frac{\partial \eta_{0}}{\partial \Omega} \mathbf{k} \cdot \frac{\partial \overline{\mathbf{u}}}{\partial T}
$$

Thus the forcing term is due to the necessity of the zeroth-order wavefield to adjust to the changing mean flow. Since $\eta_{1} \approx \eta-\eta_{0}=\Delta \eta$ from the Müller theory, the forcing term in (5.24) may be written

$$
-\frac{\partial \eta_{0}}{\partial T} \approx \frac{\Delta \eta}{T_{M}}
$$

The interaction (5.24) is essentially the Müller interaction with the perturbation $\Delta \eta$ replaced by the deviation from the flexible equilibrium state. Comparing (5.26) with the similar term (5.8) from the Müller theory,

$$
\frac{\eta-\eta_{0}}{\tau} \approx \frac{\Delta \eta}{\tau}
$$

we find that the forcing term is smaller by a factor of $T_{M} / \tau \approx 50$. The interaction described by (5.24) results in stresses a factor of 50 smaller than those predicted by the Müller theory. This is because the equilibrium state is allowed to vary with the mean flow, so the deviations from equilibrium are much smaller.

\section{d. Remarks}

The preceding theoretical arguments are not meant to be a replacement for the Müller theory. They hold true for one particular relaxation mechanism, and were meant only to demonstrate in concrete terms the possibility of a flexible equilibrium spectrum. This noninteracting property may not hold true for all relaxation mechanisms which act on the internal wavefield. A perhaps more likely reason for the apparent absence of interaction in the observational data is the extremely short relaxation times predicted by McComas and Bretherton (1977). These lead to a much weaker interaction than originally predicted by Müller. It is possible that the interaction does occur but is too weak to be observed.

However, the idea of a fiexible equilibrium state for the wavefield is not so speculative as it may seem at first sight. The "principle of detailed balance" in the kinetic theory of gases states (when translated to the wave problem) that the spectrum is in equilibrium when the net effects of the source mechanism cancel. This implies a separate balance in the radiation balance equation

$$
\left.\begin{array}{l}
\mathscr{L}(n)=0 \\
\mathscr{S}(n)=0
\end{array}\right\} .
$$

Müller and Olbers (1975) have suggested this as an approximate balance for the internal wavefield. It is also the balance assumed by the flexible equilibrium solution (5.17). For a steady mean flow, the equilibrium solution to $(5.28)$ is

$$
\eta(\mathbf{k}, \mathbf{x})=\eta[\Omega(\mathbf{k}, \mathbf{x})] .
$$

An equilibrium state of this form is consistent with free propagation of internal waves. It was shown in (5.19) that this equilibrium state cannot interact with a mean vertical shear.

As discussed by Müller and Olbers, a consequence of the separate balance in (5.28) is that the waves appear to be propagating freely, and so the wave field energy levels and length scales will vary according to the WKB approximation. This is true of the equilibrium forms (5.17) and (5.29) [transforming $\eta$ to $E\left(k_{1}, k_{2}, \omega, x_{3}\right)$ shows this], and has been observed in oceanic internal wave spectra (Leaman, 1976; Briscoe, 1975).

Another point about the diffusive equilibrium solution (5.17) is that for zero mean flow it reduces to the diffusive equilibrium solution of McComas and Bretherton [1977, Eq. (25)]. Using the dispersion relation $(5.10)$, the solution becomes

$$
\eta=C+D N\left(k_{1}{ }^{2}+k_{2}{ }^{2}\right)^{1 / 2} k_{3}{ }^{-1} .
$$

As pointed out by McComas and Bretherton, this form is realistic in that it matches the high wavenumber region of the empirical GM75 and GM76 spectra.

Thus, a flexible equilibrium state such as (5.17) is plausible for the three following reasons:

1) It is related to the "principle of detailed balance" which implies the separate balance (5.28) suggested by Müller and Olbers.

2) The separate balance implies that the equilibrium spectrum should scale in the WKB manner, as the oceanic spectrum has been observed to do.

3) The high wavenumber regions of the GM75 and GM76 spectra are close to the form described by the equilibrium spectrum.

It is not clear that the oceanic equilibrium spectrum must be flexible - only that it might be. Since a change in the form of the relaxation mechanism changes entirely the predicted strength of the wave-mean flow interaction, it is important to understand the mechanisms which shape the equilibrium wavefield. 


\section{Conclusions}

The primary conclusion to come out of this work is a negative one: the interaction between the internal wavefield and the mean vertical shear flow is very weak, a factor of about 30 weaker than the theoretical prediction of Müller (1976). The estimated wavefield Reynolds stresses at all four moorings analyzed indicated that the vertical eddy viscosity was less than $200 \mathrm{~cm}^{2} \mathrm{~s}^{-1}$ in magnitude. At two of these moorings, the conditions for validity of the Müller theory were satisfied, and the wave stresses should have been clearly observable under the conditions of measurement. The stresses were much smaller than predicted, however, and not correlated with the mean shear. The observed changes in energy level were consistent with the findings of a very weak interaction.

Theoretical investigation of the interaction of the internal wavefield with a steady vertical shear flow demonstrated that it is possible for the wavefield to have an equilibrium, steady state which is consistent with free vertical propagation of the waves, and so gives many of the WKB characteristics [strong inertial peak, change in energy level with $N(z)$ ] which have been observed. This equilibrium state is free to distort with the wavefield characteristics, and except for critical layer effects, does not exchange momentum with the shear. This is in contrast to the equilibrium state pictured in the Müller theory, in which the wavefield attempts to return to its old, nonshear, state and in so doing, exchanges momentum very strongly with the mean flow. The only interaction of our equilibrium state with the mean shear comes in through the effects of critical layer absorption. The maximum expected strength of this effect was estimated, and found to be (for a $400 \mathrm{~m}$ thick shear zone) roughly equivalent to a vertical eddy viscosity of $-100 \mathrm{~cm}^{2} \mathrm{~s}^{-1}$. No evidence to support the hypothesis of critical layer absorption was found in the observations.

No conclusions could be drawn about the horizontal eddy viscosity aspects of the Müller theory; the mechanisms which shape the spectrum are probably different in the horizontal and vertical wavenumber directions (note that all the $\mathrm{MB}$ interaction mechanisms act in the vertical wavenumber direction), and so the nature of the crucial relaxation processes is likely to be different. Another difference between the horizontal and vertical directions is that, to a large degree, the wavefield is forced at the top or bottom boundary in a horizontally homogeneous fashion, and the propagation effects most strongly felt by the wavefield are in the vertical direction. Horizontally homogeneous forcing of a horizontally inhomogeneous medium will lead to horizontal energy and momentum transfers.

At the two moorings with more energetic low- frequency fields, significant correlations of the wave stresses and energy levels with the mean flow were observed. These were inconsistent with ideas of a strictly local "eddy viscosity" type of interaction, involving primarily waves from the equilibrium spectrum. The correlations of energy and stress were instead consistent with generation of short internal waves of intense shear in the region of the main thermocline at $\sim 800 \mathrm{~m}$. When the large-scale shear $\geq 2 \times 10^{-4} \mathrm{~s}^{-1}$, the observed stresses are roughly proportional to the shear, resulting in an inferred vertical eddy viscosity of $\sim 100 \mathrm{~cm}^{2} \mathrm{~s}^{-1}$. This value for the eddy viscosity may be underestimated by up to a factor of 2 because of error in the shear estimation, and the tendency for the wavefield to become vertically symmetric (McComas and Bretherton, 1977) which is not considered here.

So we may conclude that, on the average, the mean flow is a source for internal wave energy, but a relatively minor one. From a vertical eddy viscosity of $100 \mathrm{~cm}^{2} \mathrm{~s}^{-1}$, and a shear of $2 \times 10^{-4} \mathrm{~s}^{-1}$ over the $400 \mathrm{~m}$ of the main thermocline, we estimate a depth-integrated energy input to the internal wavefield of $0.16 \mathrm{erg} \mathrm{cm}^{-2} \mathrm{~s}^{-1}$, or $\sim 16 \%$ of the tidal energy input estimated by Bell (1975). The dominant source (if there is one) has not been discovered. Experiments will have to gravitate to more likely source sites, such as the top, bottom and edges of the waveguide.

Acknowledgment. This work was performed under U. S. Office of Naval Research Contract N 00014-76-C-0197 NR 083-400.

\section{APPENDIX}

\section{Errors in Wave Stress Estimates}

The full heat equation is

$$
\begin{aligned}
\frac{\partial T}{\partial t}+\overline{\mathbf{u}} \cdot \nabla_{H} T+ & \mathbf{u} \cdot \nabla_{H} \bar{T}+\Gamma u_{3} \\
& +(\mathbf{u} \cdot \nabla T-\overline{u \cdot \nabla T})=K \nabla^{2} T
\end{aligned}
$$

The approximate size of the neglected terms will now be estimated.

We substitute "typical" wave dependence expi(k.x $-\omega t)$, multiply the equation by $u_{1}$, and rearrange, denoting $\zeta=T / \Gamma$, to obtain

$$
\begin{gathered}
\overline{u_{1} u_{3}}=i \omega \overline{u_{1} \zeta}-i(\mathbf{k} \cdot \overline{\mathbf{u}}) \overline{u_{1} \zeta} \\
-\frac{f}{N^{2}} \overline{u_{1}^{2}} \frac{\partial \bar{u}_{2}}{\partial x_{3}}+\frac{f}{N^{2}} \overline{u_{1} u_{2}} \frac{\partial \bar{u}_{1}}{\partial x_{3}} \\
-\left[k_{1} \overline{u_{1}^{2} \zeta}+k_{3} \overline{u_{1} u_{3} \zeta}\right]+\kappa k_{3}^{2} \overline{\zeta u}_{1} .
\end{gathered}
$$

It has been assumed that the mean flow is geo- 
strophic, and the mean isotherms and isopycnals are parallel, so that

$$
\frac{\partial \bar{T}}{\partial x_{\alpha}}=\Gamma \epsilon_{\alpha \beta 3} \frac{f}{N^{2}} \frac{\partial \bar{u}_{\beta}}{\partial x_{3}} .
$$

"Typical" internal wave values substituted for the terms in Eq. A2 above are as follows:

$$
\begin{aligned}
u_{1} & =5 \mathrm{~cm} \mathrm{~s}^{-1}, \quad N=2 \mathrm{cph} \\
u_{3} & =0.1 \mathrm{~cm} \mathrm{~s}^{-1}, \quad \frac{f}{N}=1 / 50 \\
\zeta & =7 \times 10^{2} \mathrm{~cm}, \quad \kappa=1.4 \times 10^{-3} \mathrm{~cm}^{2} \mathrm{~s}^{-1} \\
k_{1} & =8 \times 10^{-6} \mathrm{~cm}^{-1} \quad \text { bandwidths from } \\
k_{3} & =1.2 \times 10^{-4} \mathrm{~cm}^{-1} \quad \text { Müller (1976) }
\end{aligned}
$$

Starting with term 6 , we will now estimate the size of the neglected terms, and compare them with a typical stress magnitude of $0.1 \mathrm{~cm}^{2} \mathrm{~s}^{-2}$.

Term 6 measures the effect of molecular diffusion of heat on the stress estimates. Direct substitution gives

$$
\text { term } 6 \approx 6 \times 10^{-10} \mathrm{~cm}^{2} \mathrm{~s}^{-2} .
$$

This term is miniscule.

The effect of wavefield nonlinearity is measured by term 5. If we define $R_{3}=\overline{u_{1} u_{3} \zeta} /\left(\overline{u_{1}^{2}} \overline{u_{3}^{2}} \bar{\zeta}^{2}\right)^{1 / 2}$ as the triple correlation coefficient, then

$$
\text { term } \begin{aligned}
5 & \approx R_{3}\left|u_{1}\right|\left|u_{3}\right||\zeta| R_{3} \\
& \approx 0.04 \mathrm{~cm}^{2} \mathrm{~s}^{-2} R_{3} .
\end{aligned}
$$

$R_{3}$ is zero for either a linear (Gaussian) wavefield or a horizontally isotropic one. Since the internal wavefield appears to be both of these (Briscoe, 1977; Wunsch, 1975b) we expect $R_{3}$ to be small, perhaps 0.1 . In that case, term $5 \approx 0.004 \mathrm{~cm}^{2} \mathrm{~s}^{-2}$, a negligible effect. Note that if the wavefield were to become strongly nonlinear and directional, a moderate stress error could be induced.

Terms 3 and 4 arise because of horizontal advection of temperature by internal wave velocities across the tilted mean isopycnals. The terms were directly measured, assuming geostrophy, and found to be $5 \%$ or less when compared to the stress terms. However, since the errors are proportional to the mean shear, systematic errors can be induced in the eddy viscosity estimates. For term 3 we find, by direct substitution:

$$
\text { term } 3 \approx-150 \mathrm{~cm}^{2} \mathrm{~s}^{-1} \cdot \frac{\partial u_{2}}{\partial x_{3}} .
$$

This error affects the relation (if any) between $\overline{u_{1} u_{3}}$ and $\partial \bar{u}_{2} / \partial x_{3}$; it does not directly affect the eddy viscosity estimates. Because $\overline{u_{1} u_{2}}=0$ for a horizontally isotropic wavefield, term 4 should induce no systematic error. In practice, it was found that term 4 led to a change of $<6 \mathrm{~cm}^{2} \mathrm{~s}^{-1}$ in the eddy viscosity estimates.
The effects of Doppler shift, or advection by the mean flow, on the wavefield, are embodied in term 2. The relative strength of this term is

$$
\frac{\operatorname{term} 2}{\operatorname{term} 1}=\delta=\frac{\mathbf{k} \cdot \overline{\mathbf{u}}}{\omega}=\mathrm{O}\left(\frac{\bar{u}}{c}\right) .
$$

The ratio $\delta$ is exactly the parameter used by Müller (1976) in his perturbation expansion of the radiation balance equation. Müller argues that if most of the energy in the internal wavefield is confined to the first twenty modes, then the Doppler shift (and $\delta$ ) is small for $|\bar{u}| \lesssim 10 \mathrm{~cm} \mathrm{~s}^{-1}$. For small shear, the wave spectrum should not be greatly modified from its unperturbed (no shear) state. Assuming the GM76 form for the spectrum with a constant $5 \%$ vertical asymmetry $(52.5 \%$ of the total wave energy going downward, 47.5\% going upward), Ruddick (1977) estimates the size of term 2 to be:

$$
\text { term } 2 \approx-3.5 \times 10^{-4} \mathrm{~cm}^{2} \mathrm{~s}^{-1} \cdot \bar{u}_{1}
$$

This is ordinarily negligible. However, a word of caution must be added. If the observed stresses are primarily caused by "slow" waves with phase speeds comparable to $\bar{u}$, then the Doppler term can have a strong effect. Considering only terms 1 and 2 in Eq. (A2) we find

$$
F_{\alpha 3}^{M}=F_{\alpha 3}^{T} /\left(1-\frac{\mathbf{k} \cdot \overline{\mathbf{u}}}{\omega}\right)
$$

where $F_{\alpha 3}^{M}$ is the vertically-acting Reynolds stress inferred from the velocity and temperature measurements, and $F_{\alpha 3}^{T}$ is the true value. Note that the sign of the observed stress can be changed from the true value according to $\bar{u} \gtrless c$, where $c$ is the component of phase speed in the direction of the mean flow. If $|c|>|\bar{u}|$, or $c$ opposes $\bar{u}$, the sign is not changed. No information about the Doppler shift is available from the data presented here.

In addition to the heat equation errors were three effects related to errors in estimating the local temperature gradient in Eq. (3.1). The error in estimating the mean temperature gradient is about $\pm 10 \%$, inducing a corresponding error in the stress estimates. The effect of large-scale thermocline curvature which is advected vertically by the wave motions appears as a time oscillating perturbation to the temperature gradient. The stress error due to this is estimated to be

$$
\delta F \approx 0.014 \mathrm{~cm}^{2} \mathrm{~s}^{-2} \cdot R_{3} \approx 0.0014 \mathrm{~cm}^{2} \mathrm{~s}^{-2} \text {. }
$$

Vertical advection of a jagged temperature profile past the temperature sensor induces higher frequency temperature "noise". The velocity shear is expected to have a similar, correlated microstructure, leading to error in the stress estimates. It is argued in Ruddick (1977) that the stress error due to microstructure effects is much less than $0.04 \mathrm{~cm}^{2} \mathrm{~s}^{-2}$, but not enough is known about 
TABLE 1. Stress error estimates (one standard deviation).

\begin{tabular}{|c|c|c|}
\hline Effect & $\begin{array}{l}\text { Random } \\
\text { error }\end{array}$ & $\begin{array}{c}\text { Systematic } \\
\text { error } \\
\text { (propor- } \\
\text { tional to } \\
\partial \bar{u} / \partial x_{3} \text { ) }\end{array}$ \\
\hline Statistical error & $0.06 \mathrm{~cm}^{2} \mathrm{~s}^{-2}$ & \\
\hline Molecular diffusion of heat & $6 \times 10^{-10} \mathrm{~cm}^{2} \mathrm{~s}^{-2}$ & \\
\hline Wavefield nonlinearity & $0.004 \mathrm{~cm}^{2} \mathrm{~s}^{-2}$ & \\
\hline $\begin{array}{l}\text { Wave advection across } \\
\text { tilted isopycnals }\end{array}$ & & $\begin{array}{c}10 \mathrm{~cm}^{2} \mathrm{~s}^{-1} \\
\partial \bar{u} / \partial x_{3}\end{array}$ \\
\hline $\begin{array}{l}\text { Doppler shift by mean-flow } \\
\text { (Have assumed } \partial \bar{u} / \partial x_{3} \\
\approx \bar{u} / 500 \mathrm{~m})\end{array}$ & & $\begin{array}{c}15 \mathrm{~cm}^{2} \mathrm{~s}^{-1} \\
\partial \bar{u} / \partial x_{3}\end{array}$ \\
\hline Mooring motion & & $\begin{array}{c}5 \mathrm{~cm}^{2} \mathrm{~s}^{-1} \\
\partial \bar{u} / \partial x_{3}\end{array}$ \\
\hline Thermocline curvature & $0.0014 \mathrm{~cm}^{2} \mathrm{~s}^{-2}$ & \\
\hline $\begin{array}{l}\text { Error in temperature } \\
\text { gradient estimate }\end{array}$ & $0.005 \mathrm{~cm}^{2} \mathrm{~s}^{-2}$ & \\
\hline $\begin{array}{l}\text { Finestructure contamina- } \\
\text { tion }\end{array}$ & $0.04 \mathrm{~cm}^{2} \mathrm{~s}^{-2}$ & \\
\hline $\begin{array}{l}\text { Total (The various contribu- } \\
\text { tions are assumed } \\
\text { independent) }\end{array}$ & $0.08 \mathrm{~cm}^{2} \mathrm{~s}^{-2}$ & $\begin{array}{c}19 \mathrm{~cm}^{2} \mathrm{~s}^{-1} \\
\partial \bar{u} / \partial x_{3}\end{array}$ \\
\hline
\end{tabular}

oceanic microstructure to enable a more accurate estimate. Microstructure contamination is felt not to be a problem, but cannot yet be given a clean bill of health.

The effect of finite current meter rotor "stall speed" was also considered in Ruddick (1977). When the true current velocity is below the stall speed, $u_{s}\left(\sim 2 \mathrm{~cm} \mathrm{~s}^{-1}\right)$, the rotor doesn't turn, and the measured speed is set to $u_{s}$. This causes a small error in velocity measurement. A "worst case" estimate of the error spectrum showed that for $\bar{u} \sim 5 \mathrm{~cm} \mathrm{~s}^{-1}$, the error is only comparable to the signal in the frequency range of $f-2 f$, and is negligible at higher frequencies. Therefore, the continuum band velocity measurements are not affected. For higher mean velocities, the speed rarely drops below stall speed, and the error is negligible at all frequencies. Also, since the current error is uncorrelated with the temperature signal, the stress estimates are negligibly affected by this error.

The final source of error considered was the effect of movement of the mooring in response to ocean currents. The vertical motions of the moorings were monitored through pressure recorders, so the effects of mooring motion could be, and were, corrected. At moorings 543 and 545, where $\bar{u}$ $\approx 5 \mathrm{~cm} \mathrm{~s}^{-1}$, the correction was found to be totally unnecessary, as the mooring motion was $1 \mathrm{~m}$ or less at internal wave frequencies. At the other two moorings, with $\bar{u} \sim 30 \mathrm{~cm} \mathrm{~s} ~^{-1}$, the corrections were sizeable, and altered the eddy viscosity estimates to be presented in Section 4 by up to \pm 33 $\mathrm{cm}^{2} \mathrm{~s}^{-1}$. Assuming (conservatively) that the correction was only $85 \%$ accurate, the stress error is then about $\pm 5 \mathrm{~cm}^{2} \mathrm{~s}^{-1} \cdot \partial \bar{u}_{1} / \partial x_{3}$ or less.

The various systematic and random stress errors have been listed in Table 1 to summarize the error analysis. The estimated random errors sum (in a mean square sense) up to $0.08 \mathrm{~cm}^{2} \mathrm{~s}^{-2}$, about twice the observed stress standard deviation. The systematic errors could affect the eddy viscosity estimates by $\pm 38 \mathrm{~cm}^{2} \mathrm{~s}^{-1}$. (Two standard deviations) Thus only stress observations with statistically significant regressions and estimated eddy viscosities $\mathrm{O}\left(100 \mathrm{~cm}^{2} \mathrm{~s}^{-1}\right)$ or greater can be considered as significantly different from zero.

\section{REFERENCES}

Bell, T. H., 1975: Topographically generated internal waves in the open ocean. J. Geophys. Res., 80, 320-327.

Booker, J. R., and F. P. Bretherton, 1967: The critical layer for internal gravity waves in a shear flow. J. Fluid Mech., 27, 513-539.

Bretherton, F. P., 1969a: Momentum transport by gravity waves. Quart. J. Roy. Meteor. Soc., 95, 213-243.

- 1969 b: Waves and turbulence in stably stratified fluids. Radio Sci., 4, 1279-1287.

- , and C. J. R. Garrett, 1968: Wavetrains in inhomogeneous moving media. Proc. Roy. Soc. London, A302, 529-554.

Briscoe, M. G., 1975a: Internal waves in the ocean. Rev. Geophys. Space Phys., 13, 591-645.

- 1975b: Preliminary results from the trimoored internal wave experiment. J. Geophys. Res., 80, 3872-3884.

, 1977: Gaussianity of internal waves. J. Geophys. Res., 82, 2117-2126.

Cairns, J. L., and G. O. Williams, 1976: Internal wave observations from a midwater float, 2. J. Geophys. Res. 81, $1943-1950$.

Desaubies, Y. J. F., 1976: Analytical representation of internal wave spectra. J. Phys. Oceanogr., 6, 976-981.

Fofonoff, N. P., 1969: Spectral characteristics of internal waves in the ocean. Deep-Sea Res., 16, 58-71.

Frankignoul, C. L., 1974: Observed anisotropy of spectral characteristics of internal waves induced by low-frequency currents. J. Phys. Oceanogr., 4, 625-634.

, 1976: Observed interaction between oceanic internal waves and mesoscale eddies. Deep-Sea Res. 23, 805-820.

, and E. J. Strait, 1972: Correspondence and vertical propagation of the inertial-internal wave energy in the deep sea. Mem. Soc. Roy. Sci. Liège, 4, 151-161.

Garrett, C. J. R., 1968: On the interaction between internal gravity waves and a shear flow. J. Fluid Mech., 34, 711-720.

- and W. Munk, 1972: Space-time scales of internal waves. Geophys. Fluid Dyn., 2, 225-264.

_ a progress report. J. Geophys. Res., 80, 291-297.

Jenkins, G. M., and D. G. Watts, 1969: Spectral Analysis and Its Applications. Holden-Day, $525 \mathrm{pp}$.

Jones, W. L., 1967: Propagation of internal gravity waves in fluids with shear flow and rotation. J. Fluid Mech., 30, $439-448$

Leaman, K. D., 1976: Observations of vertical polarization and energy flux of near-inertial waves. J. Phys. Oceanogr. 6, 894-908. 
Lyttkens, E., 1963: Standard errors of regression co-efficients in the case of autocorrelated residuals. Time Series Analysis, Stud. Appl. Math., Amer. Math. Soc., 38-60.

Maslowe, S., 1973: Finite-amplitude Kelvin-Helmholtz billows. Bound. Layer Meteor., 5, 43-52.

MODE Group, 1978: The Mid-Ocean Dynamics Experiment. Deep-Sea Res., 25, 859-910.

Müller, P., 1976: On the diffusion of momentum and mass by internal gravity waves. J. Fluid Mech., 77, 789-823.

— 1977: Spectral features of the energy transfer between internal waves and a larger scale shear flow. Dyn. Atmos. Oceans 2, 49-72.

- , and D. J. Olbers, 1975: On the dynamics of internal waves in the deep ocean. J. Geophys. Res., 80, 3848-3860.

Murray, J., 1892: On the temperature of the salt- and freshwater Lochs of the West of Scotland at different depths and seasons during the years 1887 and 1888. Proc. Roy. Soc. Edin., 18, 139-228.

McComas, C. H., and Bretherton, F. P., 1977: Resonant interaction of oceanic internal waves. J. Geophys. Res. 82, 1397-1412.

McWilliams, J., 1972: Observations of kinetic energy correspondences in the internal wavefield. Deep-Sea Res., 19, $793-811$.
Olbers, D. J., 1975: Nonlinear energy transfer and the energy balance of the internal wave field in the deep ocean. J. Fluid Mech., 74, 375-399.

Rhines, P., 1973: Observations of the energy-containing oceanic eddies, and theoretical models of waves and turbulence. Bound. Layer Meteor., 4, 345-360.

Ruddick, B. R., 1977: Observations of interaction between the internal wavefield and low-frequency flows in the North Atlantic. Woods Hole Oceanographic Institution Tech. Rep. 77-68.

Sanford, T. B., 1975: Observations of the vertical structure of internal waves. J. Geophys. Res., 80, 3861-3871.

Scorer, R. S., 1969: Billow mechanics. Radio Sci., 4, 1299-1308.

Stewart, R. W., 1969: Turbulence and waves in a stratified atmosphere. Radio Sci., 4, 1269-1278.

Thorpe, S. A., 1975: The excitation, dissipation and interaction of internal waves in the deep ocean. J. Geophys. Res., 80, 328-338.

Wunsch, C., 1975a: Dean ocean internal waves: What do we really know? J. Geophys. Res. 80, 339-343. 1975b: Geographical variability of the internal wavefield: A search for sources and sinks. J. Phys. Oceanogr., 6, 471-485. 
MANDATORY DISTRIBUTION LIST

FOR UNCLASSIFIED TECHNICAL REPORTS, REPRINTS, AND FINAL REPORTS

PUBLISHED BY OCEANOGRAPHIC CONTRACTORS

OF THE OCEAN SCIENCE AND TECHNOLOGY DIVISION

OF THE OFFICE OF NAVAL RESEARCH

(REVISED NOVEMBER 1978)

1 Deputy Under Secretary of Defense (Research and Advanced Technology) Military Assistant for Environmental Science Room 3D129

Washington, D.C. 20301

Office of Naval Research

800 North Quincy Street

Arlington, VA 22217

3 ATTN: Code 483

1 ATTN: Code 460

2 ATTN: 102B

1 CDR J. C. Harlett, (USN) ONR Representative Woods Hole Oceanographic Inst.

Woods Hole, MA 02543

Commanding Officer Naval Research Laboratory Washington, D.C. 20375

6 ATTN: Library, Code 2627
12 Defense Documentation Center Cameron Station

Alexandria, VA 22314

ATTN: DCA

Commander

Naval Oceanographic Office

NSTL Station

Bay St. Louis, MS 39522

ATTN: Code 8100

ATTN: Code 6000

ATTN: Code 3300

1 NODC/NOAA

Code D781

Wiscons in Avenue, N.W.

Washington, D.C. 20235 
UNCLASSIFIED $10 / 79$

SECURITY CLASSIFICATION OF THIS PAGE (WhEN Dat Entorod)

\begin{tabular}{|c|c|}
\hline REPORT DOCUMENTATION PAGE & $\begin{array}{l}\text { READ INSTRUCTIONS } \\
\text { BEFORE COMPLETING FORM }\end{array}$ \\
\hline $\begin{array}{l}\text { 1. REPOAT NUMBER } \\
\text { WHOI }-79.62\end{array}$ & 3. RECIPIENT'S CATALOG NUMBER \\
\hline 4. TITLE (and Subttite) & 8. TYPE OF REPORT Q PERIOD COVERED \\
\hline OBSERVATIONS OF INTERACTION BETWEEN THE INTERNAL & Technical \\
\hline ATLANTIC & $\begin{array}{l}\text { 5. PERPORMINO ORG. REPORT NUMUER } \\
\text { WHOI CON. NO. } 4106\end{array}$ \\
\hline 7. AUTHOR(O) & 8. CONTRACT OR GRANT NUMBER(E) \\
\hline Barry R. Ruddick and Terrence M. Joyce & N00014-76-C-0197; \\
\hline $\begin{array}{l}\text { 9. PERFORMING ORGANIZATION NAME AND ADDRESS } \\
\text { Woods Hole Oceanographtc Institution } \\
\text { Woods Hole, MA } 02543\end{array}$ & $\begin{array}{l}\text { 10. PROGRAM ELEMENT, PROJECT, TASK } \\
\text { AREA E WORK UNIT NUMBERS } \\
\text { NR } 083-400\end{array}$ \\
\hline $\begin{array}{l}\text { 11. CONTROLLING OFFICE NAME AND ADDRESS } \\
\text { NORDA } \\
\text { National Space Technology Laboratory } \\
\text { Bay St. Louis, MS } 39529\end{array}$ & $\begin{array}{l}\text { 12. REPORT DATE } \\
\text { OCtObER } 1979 \\
\text { 13. NUMBER OF PAGES }\end{array}$ \\
\hline 14. MONITORING AGENCY NAME \& ADDRESS(tl dilferent from Controlling Oflice) & $\begin{array}{l}\text { 15. SECURITY CLASS. (of thio RopORE) } \\
\text { UnClaSSified } \\
\text { 15. DECLASSIFICATION/DOWNGRADING } \\
\text { SCHEDULE }\end{array}$ \\
\hline
\end{tabular}

Approved for public release; distribution unlimited.

17. DIST MIEUTION STATEMENT (Ot the abotract ontepod In Block 20, if dillesent from Repost)

18. SUPPLEMENTARY NOTES

Reprinted from "Journal of Physical Oceanography, Vol. 9, No. 3, May 1979, pp. $498-517^{\prime \prime}$

19. KEY WORDS (Continue on poverae alde if noceesery and idontly by block number)

1. Internal Waves

2. Waves/Mean Flow Interaction

3. Moored Current Meter Data

20. ADSTRACT (Continue on severae oldo if necoseary and identlsy by block number)

Refer to page 498 of reprint for abstract - extensive in length.

DD, FORM 1473 EDITION OF I WOV BS IS OBSOLETE

$S / N 0102 \cdot 014 \cdot 6601$ |

UNCLASSIFIED $\quad 10 / 79$

SECURITY CLASSIFICATION OF THIS PAGE (Whon DatG Bntered) 


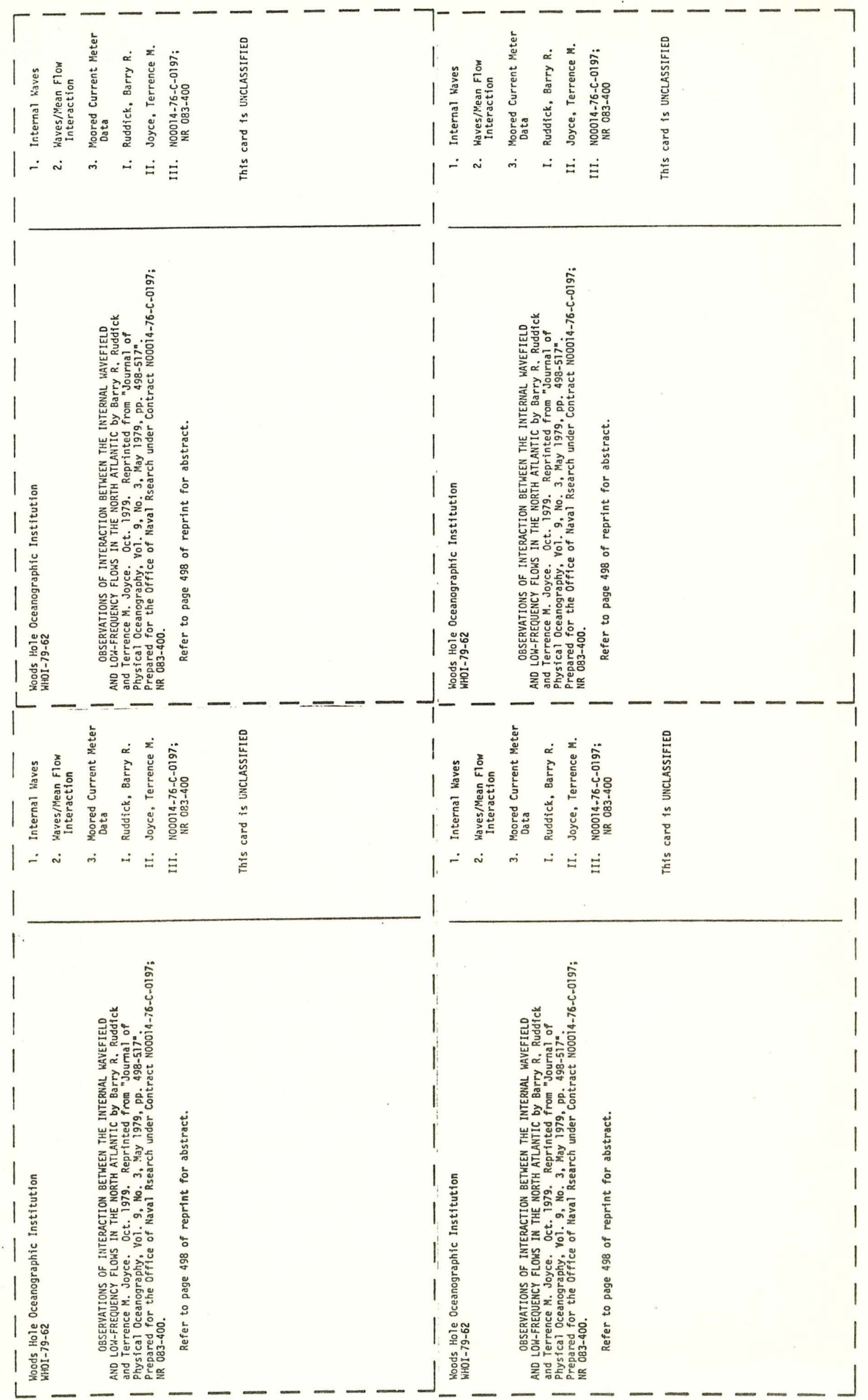

